\title{
Criteria for Three-Stage Towers of $p$-Class Fields
}

\author{
Daniel C. Mayer \\ Naglergasse 53, 8010 Graz, Austria \\ Email: quantum.algebra@icloud.com
}

How to cite this paper: Mayer, D.C. (2017) Criteria for Three-Stage Towers of p-Class Fields. Advances in Pure Mathematics, 7, 135-179.

https://doi.org/10.4236/apm.2017.72008

Received: November 24, 2016

Accepted: February 4, 2017

Published: February 7, 2017

Copyright $\odot 2017$ by author and Scientific Research Publishing Inc. This work is licensed under the Creative Commons Attribution International License (CC BY 4.0).

http://creativecommons.org/licenses/by/4.0/

\begin{abstract}
Let $p$ be a prime and $K$ be a number field with non-trivial $p$-class group $\mathrm{Cl}_{p} K$. A crucial step in identifying the Galois group $G=\mathrm{G}_{p}^{\infty} K$ of the maximal unramified pro- $p$ extension of $K$ is to determine its two-stage approximation $\mathfrak{M}=G_{p}^{2} K$, that is the second derived quotient $\mathfrak{M} \simeq G / G^{\prime \prime}$. The family $\tau_{1} K$ of abelian type invariants of the $p$-class groups $\mathrm{Cl}_{p} L$ of all unramified cyclic extensions $L / K$ of degree $p$ is called the index- $p$ abelianization data (IPAD) of $K$. It is able to specify a finite batch of contestants for the second $p$-class group $\mathfrak{M}$ of $K$. In this paper we introduce two different kinds of generalized IPADs for obtaining more sophisticated results. The multi-layered IPAD $\left(\tau_{1} K, \tau_{2} K\right)$ includes data on unramified abelian extensions $L / K$ of degree $p^{2}$ and enables sharper bounds for the order of $\mathfrak{M}$ in the case $\mathrm{Cl}_{p} K \simeq(p, p, p)$, where current implementations of the $p$-group generation algorithm fail to produce explicit contestants for $\mathfrak{M}$, due to memory limitations. The iterated IPAD of second order $\tau^{(2)} K$ contains information on non-abelian unramified extensions $L / K$ of degree $p^{2}$, or even $p^{3}$, and admits the identification of the $p$-class tower group $G$ for various infinite series of quadratic fields $K=\mathbb{Q}(\sqrt{d})$ with $\mathrm{Cl}_{p} K \simeq(p, p)$ possessing a $p$-class field tower of exact length $\ell_{p} K=3$ as a striking novelty.
\end{abstract}

\section{Keywords}

Hilbert $p$-Class Field Tower, $p$-Class Group, $p$-Principalization Types, Quadratic Fields, Unramified Cyclic Cubic Field Extensions, $p$-Class Tower Group, Relation Rank, Metabelianization, Coclass Graphs

\section{Introduction}

In a previous article [1], we provided a systematic and rigorous introduction of 
the concepts of abelian type invariants and iterated IPADs of higher order. These ideas were communicated together with impressive numerical applications at the 29th Journées Arithmétiques in Debrecen, July 2015 [2]. The purpose and the organization of the present article, which considerably extends the computational and theoretical results in [1] [2], is as follows.

Index-p abelianization data (IPADs) are explained in $\$ 2$. Our Main Theorem on three-stage towers of 3-class fields is communicated in $₫ 3$. Basic definitions concerning the Artin transfer pattern [1] [3] [4] are recalled in $\$ 4$. Then we generally put $p=3$ and consider 3 -class tower groups $(\$ 7)$. In $\$ 5$, we first restate a summary of all possible IPADs of a number field $K$ with 3-class group $\mathrm{Cl}_{3} \mathrm{~K}$ of type $(3,3)$ [[1], Thm. 3.1-3.2, pp. 290-291] in a more succinct and elegant form avoiding infinitely many exceptions, and emphasizing the role of two distinguished components, called the polarization and co-polarization, which are crucial for proving the finiteness of the batch of contestants for the second 3-class group $\mathfrak{M}=\mathrm{G}_{3}^{2} K$. Up to now, this is the unique situation where all IPADs can be given in a complete form, except for the simple case of a number field $K$ with 2-class group $\mathrm{Cl}_{2} K$ of type (2,2) [[5], $\$ 9$, pp. 501-503]. We characterize all relevant finite 3-groups by IPADs of first and second order in $\$ \$ 7.1,7.3,7.6,7.9$. These groups constitute the candidates for 3-class tower groups $\mathrm{G}_{3}^{\infty} K$ of quadratic fields $K=\mathbb{Q}(\sqrt{d})$ with 3-class group $\mathrm{Cl}_{3} K$ of type $(3,3)$. In $\$ 7.2$, results for the dominant scenario with 3-principalization $\varkappa_{1} K$ of type a are given. In $\$ \$ 7.5,7.8$, we provide evidence of unexpected phenomena revealed by real quadratic fields $K$ with types $\varkappa_{1} K$ in Scholz and Taussky's section E [[6], p. 36]. Their 3-class tower can be of length $2 \leq \ell_{3} K \leq 3$ and a sharp decision is possible by means of iterated IPADs of second order. We point out that imaginary quadratic fields with type E must always have a tower of exact length $\ell_{3} K=3$ [3] [7]. In $\$ \$ 7.10,7.11$, resp. $\$ \$ 7.12,7.13$, results for quadratic fields $K$ with 3-principalization type H.4, $\varkappa_{1} K \sim(4111)$, resp. G.19, $\varkappa_{1} K \sim(2143)$, are proved.

In the last section $\$ 8$ on multi-layered IPADs, it is our endeavour to point out that the rate of growth of successive derived quotients $G_{p}^{n} K \simeq G / G^{(n)}, n \geq 2$, of the $p$-class tower group $G=G_{p}^{\infty} K$ is still far from being known for imaginary quadratic fields $K$ with $p$-class rank $\rho \geq 3$, where the criterion of Koch and Venkov [8] ensures an infinite $p$-class tower with $\ell_{p} K=\infty$.

\section{Index- $p$ Abelianization Data}

Let $p$ be a prime number. According to the Artin reciprocity law of class field theory [9], the unramified cyclic extensions $L / K$ of relative degree $p$ of a number field $K$ with non-trivial $p$-class group $\mathrm{Cl}_{p} K$ are in a bijective correspondence to the subgroups of index $p$ in $\mathrm{Cl}_{p} K$. Their number is given by $\frac{p^{\varrho}-1}{p-1}$ if $\varrho$ denotes the $p$-class rank of $K \quad[[10]$, Thm. 3.1]. The reason for this fact is that the Galois group $\mathrm{G}_{p}^{1} K:=\mathrm{Gal}\left(\mathrm{F}_{p}^{1} K / K\right)$ of the maximal unramified abelian $p$-extension $\mathrm{F}_{p}^{1} K / K$, which is called the first Hilbert $p$ - 
class field of $K$, is isomorphic to the $p$-class group $\mathrm{Cl}_{p} K$. The fields $L$ are

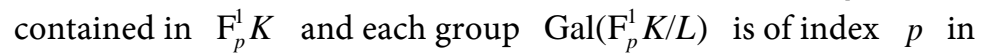
$\mathrm{G}_{p}^{1} K \simeq \mathrm{Cl}_{p} K$.

It was also Artin's idea [11] to leave the abelian setting of class field theory and to consider the second Hilbert $p$-class field $\mathrm{F}_{p}^{2} K=\mathrm{F}_{p}^{1}\left(\mathrm{~F}_{p}^{1} K\right)$, that is the maximal unramified metabelian $p$-extension of $K$, and its Galois group $\mathfrak{M}:=\mathrm{G}_{p}^{2} K:=\mathrm{Gal}\left(\mathrm{F}_{p}^{2} K / K\right)$, the so-called second $p$-class group of $K[[5][6]$, p. 41], for proving the principal ideal theorem that $\mathrm{Cl}_{p} K$ becomes trivial when it is extended to $\mathrm{Cl}_{p}\left(\mathrm{~F}_{p}^{1} K\right)$ [12]. Since $K \leq L \leq \mathrm{F}_{p}^{1} K \leq \mathrm{F}_{p}^{1} L \leq \mathrm{F}_{p}^{2} K$ is a nondecreasing tower of normal extensions for any assigned unramified abelian $p$ extension $L / K$, the $p$-class group of $L$, $\mathrm{Cl}_{p} L \simeq \operatorname{Gal}\left(\mathrm{F}_{p}^{1} L / L\right) \simeq \operatorname{Gal}\left(\mathrm{F}_{p}^{2} K / L\right) / \operatorname{Gal}\left(\mathrm{F}_{p}^{2} K / \mathrm{F}_{p}^{1} L\right)$, is isomorphic to the $a b$ elianization $H / H^{\prime}$ of the subgroup $H:=\mathrm{Gal}\left(\mathrm{F}_{p}^{2} K / L\right)$ of the second $p$-class group $\mathrm{Gal}\left(\mathrm{F}_{p}^{2} K / K\right)$ which corresponds to $L$ and whose commutator subgroup is given by $H^{\prime}=\mathrm{Gal}\left(\mathrm{F}_{p}^{2} K / \mathrm{F}_{p}^{1} L\right)$.

In particular, the structure of the $p$-class groups $\mathrm{Cl}_{p} L$ of all unramified cyclic extensions $L / K$ of relative degree $p$ can be interpreted as the abelian type invariants of all abelianizations $H / H^{\prime}$ of subgroups $H=\operatorname{Gal}\left(\mathrm{F}_{p}^{2} K / L\right)$ of index $p$ in the second $p$-class group $\operatorname{Gal}\left(\mathrm{F}_{p}^{2} K / K\right)$, which has been dubbed the index- $p$ abelianization data, briefly IPAD, $\tau_{1} K$ of $K$ by Boston, Bush, and Hajir [13]. This kind of information would have been incomputable and thus useless about twenty years ago. However, with the availability of computational algebra systems like PARI/GP [14] and MAGMA [15] [16] [17] it became possible to compute the class groups $\mathrm{Cl}_{p} L$, collect their structures in the IPAD $\tau_{1} K$, reinterpret them as abelian quotient invariants of subgroups $H$ of $\mathrm{G}_{p}^{2} \mathrm{~K}$, and to use this information for characterizing a batch of finitely many $p$-groups, occasionally even a unique $p$-group, as contestants for the second $p$-class group $\mathfrak{M}=\mathrm{G}_{p}^{2} K$ of $K$, which in turn is a two-stage approximation of the (potentially infinite) pro- $p$ group $G:=\mathrm{G}_{p}^{\infty} K:=\mathrm{Gal}\left(\mathrm{F}_{p}^{\infty} K / K\right)$ of the maximal unramified pro- $p$ extension $\mathrm{F}_{p}^{\infty} K$ of $K$, that is its Hilbert $p$ class tower.

As we proved in the main theorem of [[4], Thm. 5.4], the IPAD is usually unable to permit a decision about the length $\ell:=\ell_{p} K$ of the $p$-class tower of $K$ when non-metabelian candidates for $\mathrm{G}_{p}^{\infty} K$ exist. For solving such problems, iterated IPADs $\tau^{(2)} K$ of second order are required.

\section{The $p$-Principalization Type}

Until very recently, the length $\ell$ of the $p$-class tower

$$
K<\mathrm{F}_{p}^{1} K<\mathrm{F}_{p}^{2} K<\cdots \mathrm{F}_{p}^{\ell} K=\mathrm{F}_{p}^{\ell+1} K=\cdots=\mathrm{F}_{p}^{\infty} K
$$

over a quadratic field $K=\mathbb{Q}(\sqrt{d})$ with $p$-class rank $\varrho=2$, that is, with $p$ class group $\mathrm{Cl}_{p} K$ of type $\left(p^{u}, p^{v}\right), u \geq v \geq 1$, was an open problem. Apart from the proven impossibility of an abelian tower with $\ell=1$ [[5], Thm. 4.1.(1)], it was unknown which values $\ell \geq 2$ can occur and whether $\ell=\infty$ is possible 
or not. In contrast, it is known that $\ell=1$ for any number field $K$ with $p$ class rank $\varrho=1$, i.e., with non-trivial cyclic $p$-class group $\mathrm{Cl}_{p} K$, and that $\ell=\infty$ for an imaginary quadratic field with $p$-class rank $\varrho \geq 3$, when $p$ is odd [8].

The finite batch of contestants for $\mathfrak{M}=\mathrm{G}_{p}^{2} K$, specified by the IPAD $\tau_{1} K$, can be narrowed down further if the $p$-principalization type of $K$ is known. That is the family $\varkappa_{1} K$ of all kernels $\operatorname{ker} T_{K, L}$ of $p$-class transfers $T_{K, L}: \mathrm{Cl}_{p} K \rightarrow \mathrm{Cl}_{p} L$ from $K$ to unramified cyclic superfields $L$ of degree $p$ over $K$. In view of the open problem for the length of the $p$-class tower, there arose the question whether each possible $p$-principalization type $\varkappa_{1} K$ of a quadratic field $K$ with $\mathrm{Cl}_{p} K$ of type $(p, p)$ is associated with a fixed value of the tower length $\ell_{p} K$.

For $p=3$ and $\mathrm{Cl}_{3} K$ of type (3,3), there exist 23 distinct 3-principalization types [[18], Tbl.6-7], designated by X.n, where $X$ denotes a letter in $\{A, D, E, F, G, H, a, b, c, d\}$ and $n$ denotes a certain integer in $\{1, \cdots, 25\}$, more explicitly:

A.1, D.5, D.10, E.6, E.8, E.9, E.14, F.7, F.11, F.12, F.13, G.16, G.19, H.4, a.1, a.2, a.3, b.10, c.18, c.21, d.23, d.25.

In this article, we establish the last but one step for the proof of the following solution to the open problem for $p=3$ and quadratic fields $K$ with $\mathrm{Cl}_{3} K \simeq(3,3)$.

Theorem 3.1. (Main theorem on the length of the 3-class tower for 3-class rank two)

1) For each of the 13 types of 3-principalization X.n with upper case letter $\mathrm{X} \neq \mathrm{A}$, there exists an imaginary quadratic field $K=\mathbb{Q}(\sqrt{d}), d<0$, of that type such that $2 \leq \ell_{3} K \leq 3$.

2) For each of the 22 types of 3-principalization $X . n \neq A .1$, there exists a real quadratic field $K=\mathbb{Q}(\sqrt{d}), d>0$, of that type such that $2 \leq \ell_{3} K \leq 3$.

Remark 3.1. Type A.1 must be excluded for quadratic base fields $K$, according to [[5], Cor. 4.2]. It occurs, however, with $\ell=2$ for cyclic cubic fields with two primes dividing the conductor [19].

Concerning the steps for the proof, we provide information in the form of Table 1. An asterisk indicates the present paper. The last step has been completed in collaboration with M. F. Newman but has not been published yet [20]. Only the types G.16 and G.19 must be distinguished by their integer identifier, otherwise the types denoted by the same letter behave completely similar. Additionally, we give the smallest logarithmic order $\operatorname{lo}(G):=\log _{3}|G|$.

Remark 3.2. None of the types sets in with a length $\ell \geq 4$. Type $D$ behaves completely rigid with $\ell=2$, fixed class 3 , and coclass 2 . Type a is also confined to $\ell=2$ but admits unbounded nilpotency class with fixed coclass 1 . For type E, we have $\ell=3$ with unbounded class and coclass for imaginary fields, and the unique exact dichotomy $\ell \in\{2,3\}$ for real fields. For type $c$, the length $\ell=3$ is fixed with unbounded class and coclass for real fields. The most extensive flexibility is revealed by fields of the types F,G,H and b,d, where 
Table 1. Steps of the proof with references.

\begin{tabular}{cccccccccccc}
\hline Type & D & E & F & G.16 & G.19 & H & a & b & c & d & Base Fields \\
\hline$\ell_{3} K$ & 2 & 3 & $\geq 3$ & $\geq 3$ & $\geq 3$ & $\geq 3$ & & & & & imaginary \\
$\operatorname{lo}(G)$ & 5 & $\geq 8$ & $\geq 20$ & $\geq 11$ & $\geq 11$ & $\geq 8$ & & & & & quadratic \\
$\operatorname{Ref.}$ & {$[6]$} & {$[7]$} & {$[20]$} & {$[20]$} & $*$ & $*$ & & & & & fields \\
$\ell_{3} K$ & 2 & 2 or 3 & $\geq 3$ & $\geq 3$ & $\geq 3$ & $\geq 3$ & 2 & $\geq 3$ & 3 & $\geq 3$ & real \\
$\operatorname{lo}(G)$ & 5 & $\geq 7$ & $\geq 10$ & $\geq 9$ & $\geq 7$ & $\geq 7$ & $\geq 4$ & $\geq 10$ & $\geq 7$ & $\geq 10$ & quadratic \\
& & & & & & & & & {$[22]$} & {$[20]$} & fields \\
$\operatorname{Ref.}$ & {$[21]$} & $*$ & {$[20]$} & {$[20]$} & $*$ & $*$ & $*$ & {$[20]$} & {$[23]$} & {$[20]$} & \\
\hline
\end{tabular}

any finite unbounded length $\ell \geq 3$ can occur with variable class and coclass. We expect that an actually infinite tower with $\ell=\infty$ is impossible for $\mathrm{Cl}_{3} \mathrm{~K} \simeq(3,3)$.

\section{The Artin Transfer Pattern}

Let $p$ be a prime number and $G$ be a pro- $p$ group with finite abelianization $G / G^{\prime}$, more precisely, assume that the commutator subgroup $G^{\prime}$ is of index $\left(G: G^{\prime}\right)=p^{v}$ with an integer exponent $v \geq 0$.

Definition 4.1. For each integer $0 \leq n \leq v$, let $\operatorname{Lyr}_{n} G:=\left\{G^{\prime} \leq H \leq G \mid(G: H)=p^{n}\right\}$ be the nth layer of normal subgroups of $G$ containing $G^{\prime}$.

Definition 4.2. For any intermediate group $G^{\prime} \leq H \leq G$, we denote by $T_{G, H}: G \rightarrow H / H^{\prime}$ the Artin transfer homomorphism from $G$ to $H / H^{\prime}$ [[4], Dfn. 3.1], and by $\tilde{T}_{G, H}: G / G^{\prime} \rightarrow H / H^{\prime}$ the induced transfer.

1) Let $\tau(G):=\left[\tau_{0} G ; \cdots ; \tau_{v} G\right]$ be the multi-layered transfer target type (TTT) of $G$, where $\tau_{n} G:=\left(H / H^{\prime}\right)_{H \in \mathrm{Lyr}_{n} G}$ for each $0 \leq n \leq v$.

2) Let $\varkappa(G):=\left[\varkappa_{0} G ; \cdots ; \varkappa_{v} G\right]$ be the multi-layered transfer kernel type (TKT) of $G$, where $\varkappa_{n} G:=\left(\operatorname{ker} \tilde{T}_{G, H}\right)_{H \in \mathrm{Ly}_{n} G}$ for each $0 \leq n \leq v$.

Definition 4.3. The pair $\operatorname{AP}(G):=(\tau(G), \varkappa(G))$ is called the (restricted) Artin pattern of $G$.

Definition 4.4. The first order approximation $\tau^{(1)} G:=\left[\tau_{0} G ; \tau_{1} G\right]$ of the TTT, resp. $\varkappa^{(1)} G:=\left[\varkappa_{0} G ; \varkappa_{1} G\right]$ of the TKT, is called the index- $p$ abelianization data (IPAD), resp. index- $p$ obstruction data (IPOD), of $G$.

Definition 4.5. $\tau^{(2)} G:=\left[\tau_{0} G ;\left(\tau^{(1)} H\right)_{H \in \mathrm{Lyy}_{1} G}\right]$ is called iterated IPAD of $2^{\text {nd }}$ order of $G$.

Remark 4.1. For the complete Artin pattern $\operatorname{AP}_{c}(G)$ see [[4], Dfn. 5.3].

1) Since the 0th layer (top layer), $\operatorname{Lyr}_{0} G=\{G\}$, consists of the group $G$ alone, and $T_{G, G}: G \rightarrow G / G^{\prime}$ is the natural projection onto the commutator quotient with kernel $\operatorname{ker}\left(T_{G, G}\right)=G^{\prime}$, we usually omit the trivial top layer $\varkappa_{0} G$ and identify the IPOD $\varkappa^{(1)} G$ with the first layer $\varkappa_{1} G$ of the TKT.

2) In the case of an elementary abelianization of rank two, $\left(G: G^{\prime}\right)=p^{2}$, we 
also identify the TKT $\varkappa(G)$ with its first layer $\varkappa_{1} G$, since the 2nd layer (bottom layer), $\operatorname{Lyr}_{2} G=\left\{G^{\prime}\right\}$, consists of the commutator subgroup $G^{\prime}$ alone, and the kernel of $T_{G, G^{\prime}}: G \rightarrow G^{\prime} / G^{\prime \prime}$ is always total, that is $\operatorname{ker}\left(T_{G, G^{\prime}}\right)=G$, according to the principal ideal theorem [12].

\section{All Possible IPADs of 3 -Groups of Type $(3,3)$}

Since the abelian type invariants of certain IPAD components of an assigned 3-group $G$ depend on the parity of the nilpotency class $c$ or coclass $r$, a more economic notation, which avoids the tedious distinction of the cases odd or even, is provided by the following definition [[24], \$3].

Definition 5.1. For an integer $n \geq 2$, the nearly homocyclic abelian 3-group $\mathrm{A}(3, n)$ of order $3^{n}$ is defined by its type invariants $(q+r, q) \hat{=}\left(3^{q+r}, 3^{q}\right)$, where the quotient $q \geq 1$ and the remainder $0 \leq r<2$ are determined uniquely by the Euclidean division $n=2 q+r$. Two degenerate cases are included by putting $\mathrm{A}(3,1):=(1) \hat{=}(3)$ the cyclic group $C_{3}$ of order 3 , and $\mathrm{A}(3,0):=(0) \hat{=} 1$ the trivial group of order 1 .

In the following theorem and in the whole remainder of the article, we use the identifiers of finite 3-groups up to order $3^{8}$ as they are defined in the SmallGroups Library [25] [26]. They are of the shape <order, counter>, where the counter is motivated by the way how the output of descendant computations is arranged in the $p$-group generation algorithm by Newman [27] and O'Brien [28].

Theorem 5.1. (Complete classification of all IPADs with $\tau_{0} \simeq(3,3)$ [24]) Let $G$ be a pro-3 group with abelianization $G / G^{\prime}$ of type $(3,3)$ and metabelianization $\mathfrak{M}=G / G^{\prime \prime}$ of nilpotency class $c=\mathrm{cl}(\mathfrak{M}) \geq 2$, defect $0 \leq k=k(\mathfrak{M}) \leq 1$, and coclass $r=\operatorname{cc}(\mathfrak{M}) \geq 1$. Assume that $\mathfrak{M}$ does not belong to the finitely many exceptions in the list below. Then the IPAD $\tau^{(1)} G=\left[\tau_{0} G ; \tau_{1} G\right]$ of $G$ in terms of nearly homocyclic abelian 3-groups is given by

$$
\begin{aligned}
& \tau_{0} G=\left(1^{2}\right) ; \\
& \tau_{1} G=(\overbrace{\mathrm{A}(3, c-k)}^{\text {polarization }}, \overbrace{\mathrm{A}(3, r+1)}^{\text {co-polarization }}, T_{3}, T_{4}),
\end{aligned}
$$

where the polarized first component of $\tau_{1} G$ depends on the class $c$ and defect $k$, the co-polarized second component increases with the coclass $r$, and the third and fourth component are completely stable for $r \geq 3$ but depend on the coclass tree containing $\mathfrak{M}$ for $1 \leq r \leq 2$ in the following manner

$$
\left(T_{3}, T_{4}\right)=\left\{\begin{array}{lll}
\left(\mathrm{A}(3, r+1)^{2}\right) & \text { if } & r=2, \mathfrak{M} \in \mathcal{T}^{2}\langle 243,8\rangle \quad \text { or } \quad r=1, \\
\left(1^{3}, \mathrm{~A}(3, r+1)\right) & \text { if } \quad r=2, \mathfrak{M} \in \mathcal{T}^{2}\langle 243,6\rangle, \\
\left(\left(1^{3}\right)^{2}\right) & \text { if } \quad r=2, \mathfrak{M} \in \mathcal{T}^{2}\langle 243,3\rangle \quad \text { or } r \geq 3 .
\end{array}\right.
$$

Anomalies of finitely many, precisely 13 , exceptional groups are summarized 
in the following list.

$$
\begin{array}{ll}
\tau_{1} G=\left((1)^{4}\right) & \text { for } \mathfrak{M} \simeq\langle 9,2\rangle, c=1, r=1, \\
\tau_{1} G=\left(1^{2},(2)^{3}\right) & \text { for } \mathfrak{M} \simeq\langle 27,4\rangle, c=2, r=1, \\
\tau_{1} G=\left(1^{3},\left(1^{2}\right)^{3}\right) & \text { for } \mathfrak{M} \simeq\langle 81,7\rangle, c=3, r=1, \\
\tau_{1} G=\left(\left(1^{3}\right)^{3}, 21\right) & \text { for } \mathfrak{M} \simeq\langle 243,4\rangle, c=3, r=2, \\
\tau_{1} G=\left(1^{3},(21)^{3}\right) & \text { for } \mathfrak{M} \simeq\langle 243,5\rangle, c=3, r=2, \\
\tau_{1} G=\left(\left(1^{3}\right)^{2},(21)^{2}\right) & \text { for } \mathfrak{M} \simeq\langle 243,7\rangle, c=3, r=2, \\
\tau_{1} G=\left((21)^{4}\right) & \text { for } \mathfrak{M} \simeq\langle 243,9\rangle, c=3, r=2, \\
\tau_{1} G=\left(\left(1^{3}\right)^{3}, 21\right) & \text { for } \mathfrak{M} \simeq\langle 729,44 \cdots 47\rangle, c=4, r=2, \\
\tau_{1} G=\left((21)^{4}\right) & \text { for } \mathfrak{M} \simeq\langle 729,56 \cdots 57\rangle, c=4, r=2 .
\end{array}
$$

The polarization and the co-polarization we had in our mind when we spoke about a bi-polarization in [[29], Dfn. 3.2, p. 430]. Meanwhile, we have provided yet another proof for the existence of stable and polarized IPAD components with the aid of a natural partial order on the Artin transfer patterns distributed over a descendant tree [[4], Thm. 6.1-6.2].

Proof. Equations (5.1) and (5.2) are a succinct form of information which summarizes all statements about the first TTT layer $\tau_{1} G$ in the formulas (19), (20) and (22) of [[1], Thm. 3.2, p. 291] omitting the claims on the second TTT layer $\tau_{2} G$. Here we do not need the restrictions arising from lower bounds for the nilpotency class $c=\mathrm{cl}(\mathfrak{M})$ in the cited theorem, since the remaining cases for small values of $c$ can be taken from [[1], Thm. 3.1, p. 290], with the exception of the following 13 anomalies in formula (5.3):

The abelian group $\langle 9,2\rangle \simeq(3,3)$, the extra special group $\langle 27,4\rangle$, and the group $\langle 81,7\rangle \simeq \operatorname{Syl}_{3}\left(A_{9}\right)$ do not fit into the general rules for 3-groups of coclass 1 . These three groups appear in the top region of the tree diagram in the Figure 1 and Figure 2.

The four sporadic groups $\langle 243, n\rangle$ with $n \in\{4,5,7,9\}$ and the six sporadic groups $\langle 729, n\rangle$ with $n \in\{44, \cdots, 47,56,57\}$ do not belong to any coclass-2 tree, as shown in Figure 5, whence the conditions in Equation (5.2) cannot be applied to them.

On the other hand, there is no need to list the groups $\langle 27,3\rangle$ and $\langle 81,8 \cdots 10\rangle$ in formula (14), the groups $\langle 243, n\rangle$ with $n \in\{3,6,8\}$ in formula (15), and the groups $\langle 729, n\rangle$ with $n \in\{34, \cdots, 39\}$ in formula (16) of [[1], Thm. 3.1, p. 290], since they perfectly fit into the general pattern.

Remark 5.1. The reason why we exclude the second TTT layer $\tau_{2} G$ from Theorem 5.1, while it is part of [[1], Thm. 3.1-3.2, pp. 290-291], is that we want to reduce the exceptions of the general pattern to a finite list, whereas the irregular case of the abelian quotient invariants of the commutator subgroup $G^{\prime}$, which forms the single component of $\tau_{2} G$, occurs for each even value of 


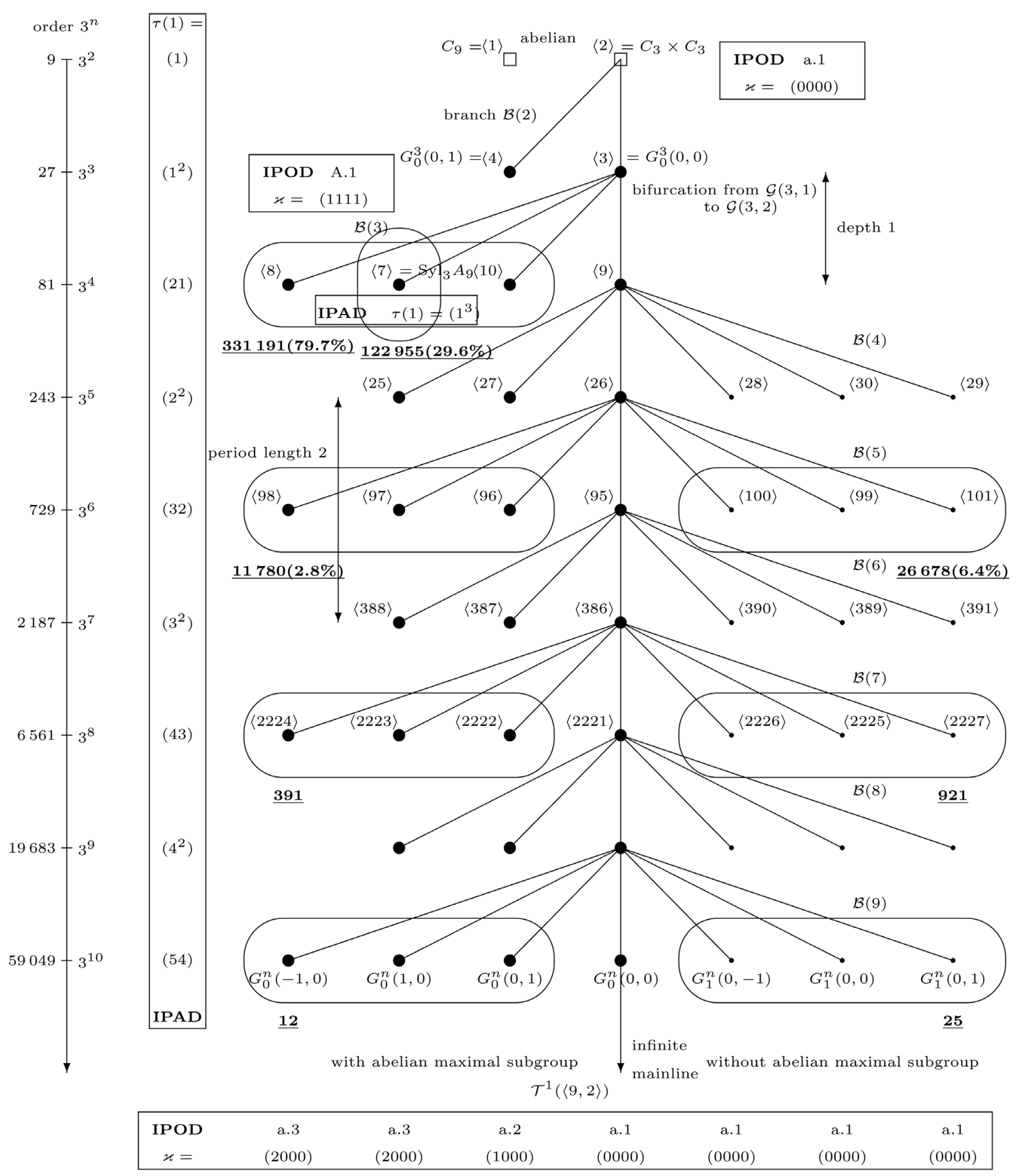

Figure 1. Distribution of absolute frequencies of $\mathrm{G}_{3}^{2} K$ on the coclass tree $\mathcal{T}^{1}\langle 9,2\rangle$.

the coclass $r=\mathrm{cc}(\mathfrak{M}) \equiv 0 \quad(\bmod 2)$ and thus infinitelyoften.

Theorem 5.2. (Finiteness of the batch of contestants for the second p-class group $\mathfrak{M}=\mathrm{G}_{p}^{2} K$ ) If $p=3, \tau_{0}=\left(1^{2}\right)$, and $\tau_{1}$ denotes an assigned family $\left(\tau_{1}(i)\right)_{1 \leq i \leq 4}$ of four abelian type invariants, then the set $\operatorname{Cnt}_{p}^{2}\left(\tau_{0}, \tau_{1}\right)$ of all (isomorphism classes of) finite metabelian $p$-groups $\mathfrak{M}$ such that 


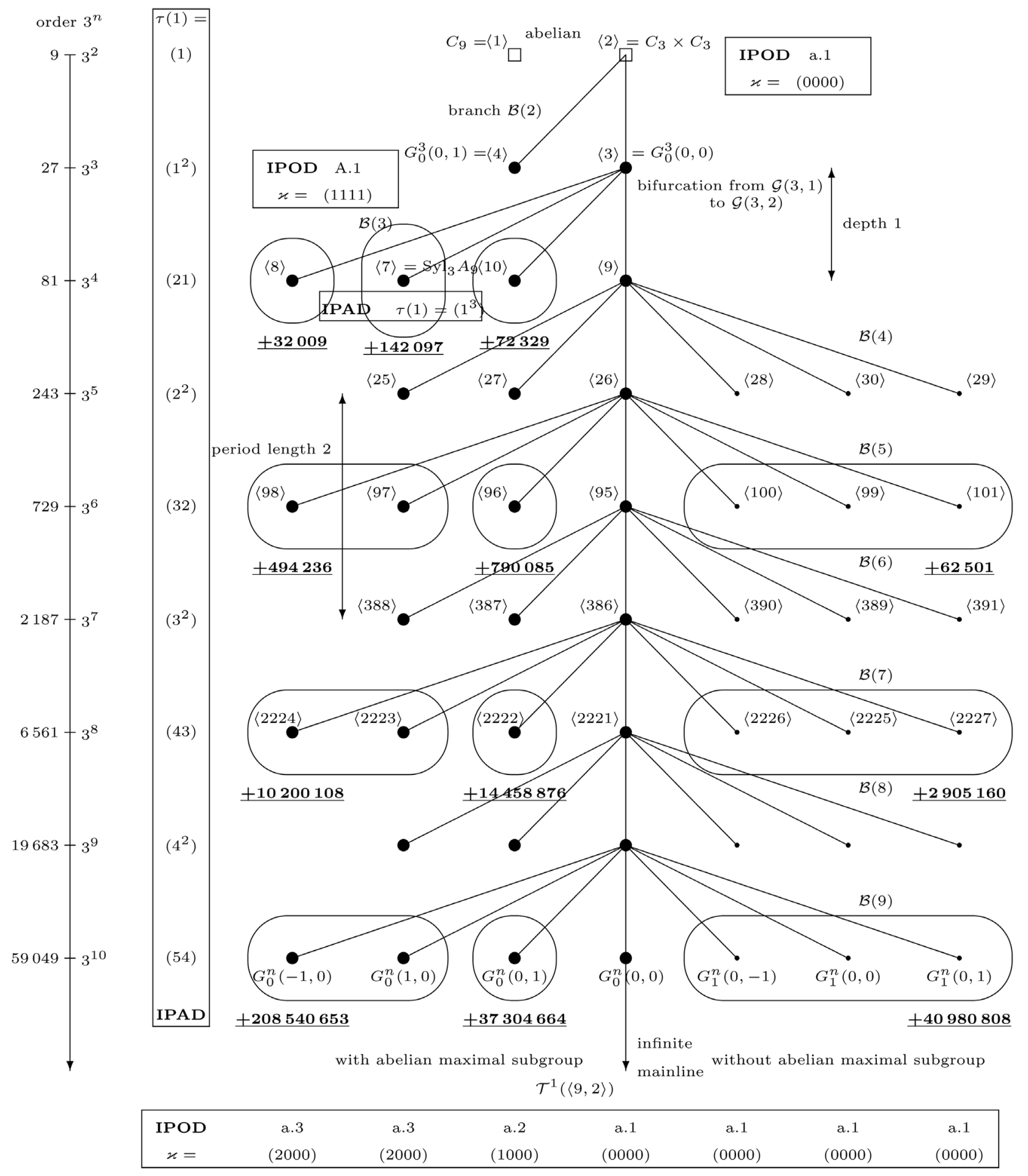

Figure 2. Distribution of minimal discriminants for $G_{3}^{2} K$ on the coclass tree $\mathcal{T}^{1}\langle 9,2\rangle$.

$\tau_{0} \mathfrak{M}=\mathfrak{M} / \mathfrak{M}^{\prime} \simeq \tau_{0}$ and $\tau_{1} \mathfrak{M}=\left(H / H^{\prime}\right)_{H \in \text { Ly } y_{1} \mathfrak{M}} \simeq \tau_{1}$ is finite

Proof. We have $\operatorname{Cnt}_{p}^{2}\left(\tau_{0}, \tau_{1}\right)=\varnothing$, when $\tau_{1}$ is malformed [[1], Dfn. 5.1, p. 294]. For $p=3$ and $\tau_{0}=\left(1^{2}\right)$, Theorem 5.1 ensures the validity of the following general Polarization Principle: There exist a few components of a non-malformed family $\tau_{1}$ which determine the nilpotency class $c:=\operatorname{cl}(\mathfrak{M})$ 
and the coclass $r:=\operatorname{cc}(\mathfrak{M})$ of a finite metabelian $p$-group $\mathfrak{M}$ with $\tau_{1} \mathfrak{M}=\left(H / H^{\prime}\right)_{H \in \mathrm{Ly}_{1} \mathfrak{M}} \simeq \tau_{1}$. Together with the Coclass Theorems [[30], $\$ 5$, p. 164, and Equation (10), p. 168], the polarization principle proves the claim.

\section{Tables and Figures of Possible 3-Groups $G_{3}^{2} K$ and $G_{3}^{\infty} K$}

\subsection{Tables}

In this article, we shall frequently deal with finite 3-groups $G$ of huge orders $|G| \geq 3^{9}$ for which no identifiers are available in the SmallGroups database [25] [26]. For instance in Table 6, and in the Figure 6 and Figure 7. A work-around for these cases is provided by the relative identifiers of the ANUPQ (Australian National University $p$-Quotient) package [31] which is implemented in our licence of the computational algebra system MAGMA [15] [16] [17] and in the open source system GAP [32].

Definition 6.1. Let $p$ be a prime number and $G$ be a finite $p$-group with nuclear rank $v \geq 1$ [[30], Equation (28), p. 178] and immediate descendant numbers $N_{1}, \cdots, N_{v}$ [[30], Equation (34), p. 180]. Then we denote the ith immediate descendant of step size $s$ of $G$ by the symbol

$$
G-\# s ; i
$$

for each $1 \leq s \leq v$ and $1 \leq i \leq N_{s}$.

Recall that a group with nuclear rank $v=0$ is a terminal leaf without any descendants.

All numerical results in this article have been computed by means of the computational algebra system MAGMA [15] [16] [17]. The p-group generation algorithm by Newman [27] and O'Brien [28] was used for the recursive construction of descendant trees $\mathcal{T}(R)$ of finite $p$-groups $G$. The tree root (starting group) $R$ was taken to be $\langle 9,2\rangle$ for Table 2 and the Figure 2, Figure 1, Figure 5, $\langle 243,6\rangle$ for Table 3 and Figure 3, $\langle 243,8\rangle$ for Table 4 and Figure 4, $\langle 243,4\rangle$ for Table 5 and Figure 6, and $\langle 243,9\rangle$ for Table 6 and Figure 7. For computing group theoretic invariants of each tree vertex $G$, we implemented the Artin transfers $T_{G, H}$ from a finite $p$-group $G$ of type $G / G^{\prime} \simeq(p, p)$ to its maximal subgroups $H \unlhd G$ in a MAGMA program script as described in [[4], §4.1].

\subsection{Figures}

Basic definitions, facts, and notation concerning descendant trees of finite $p$ groups are summarized briefly in [[29], $\$ 2$, pp. 410-411], [33]. They are discussed thoroughly in the broadest detail in the initial sections of [30]. Trees are crucial for the recent theory of $p$-class field towers [34] [35] [36], in particular for describing the mutual location of $\mathrm{G}_{3}^{2} K$ and $\mathrm{G}_{3}^{\infty} K$.

Generally, the vertices of coclass trees in the Figures 1-4, of the sporadic part of a coclass graph in Figure 5, and of the descendant trees in the Figure 6 and Figure 7 represent isomorphism classes of finite 3-groups. Two vertices are connected by a directed edge $G \rightarrow H$ if $H$ is isomorphic to the last lower 
Table 2. IPOD $\varkappa_{1} G$ and iterated IPAD $\tau_{*}^{(2)} G$ of 3-groups $G$ of coclass $\operatorname{cc}(G)=1$.

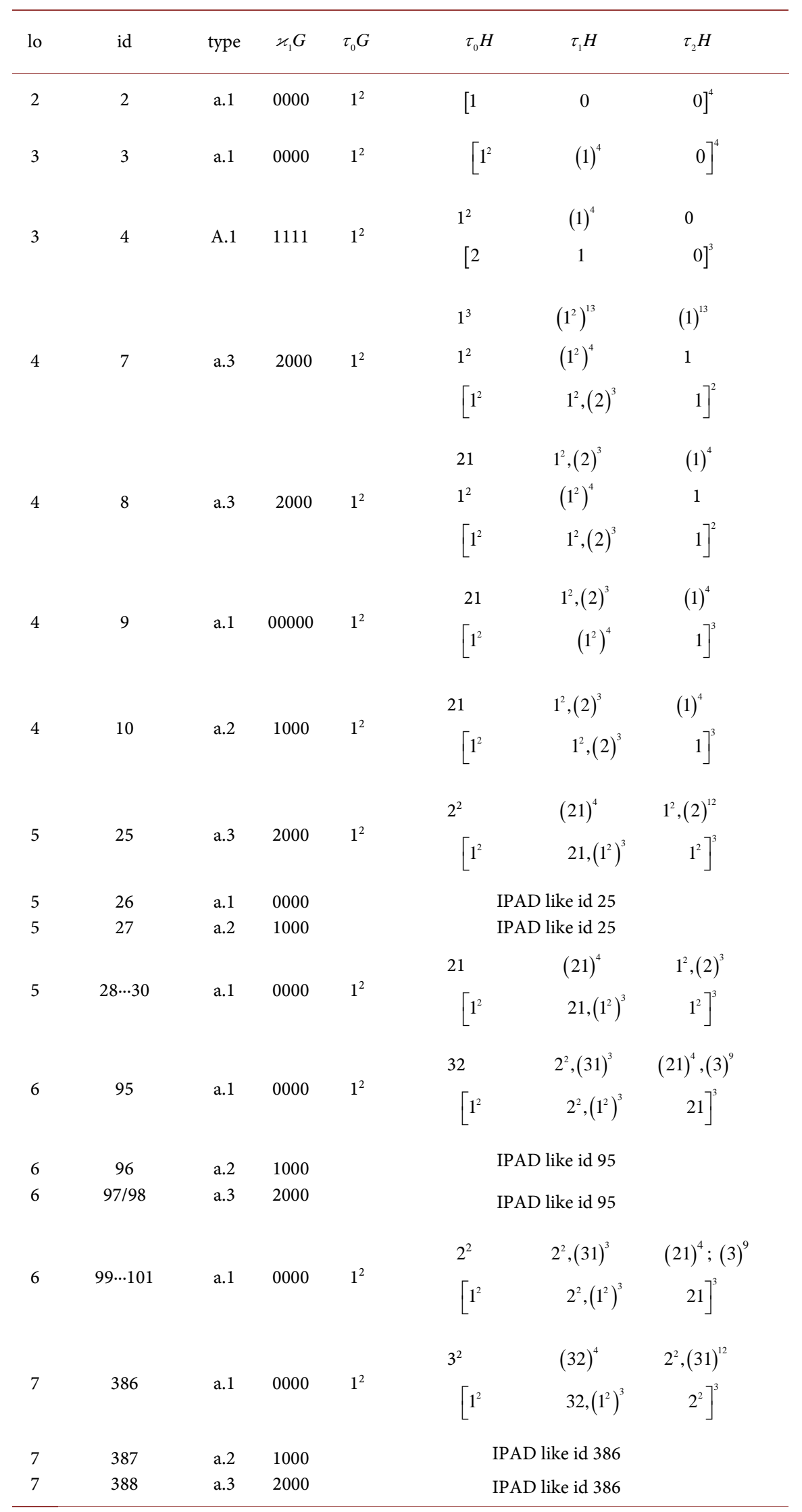




\section{Continued}

\begin{tabular}{|c|c|c|c|c|c|c|c|}
\hline 7 & $389 \cdots 391$ & a. 1 & 0000 & $1^{2}$ & $\begin{array}{l}32 \\
{\left[1^{2}\right.}\end{array}$ & $\begin{array}{l}(32)^{4} \\
32,\left(1^{2}\right)^{3}\end{array}$ & $\begin{array}{c}2^{2},(31)^{12} \\
\left.2^{2}\right]^{3}\end{array}$ \\
\hline 8 & 2221 & a.1 & 0000 & $1^{2}$ & $\begin{array}{l}43 \\
{\left[1^{2}\right.}\end{array}$ & $\begin{array}{c}3^{2},(42)^{3} \\
3^{2},\left(1^{2}\right)^{3}\end{array}$ & $\begin{array}{c}(32)^{4},(41)^{9} \\
32]^{3}\end{array}$ \\
\hline $\begin{array}{l}8 \\
8\end{array}$ & $\begin{array}{c}2222 \\
2223 / 2224\end{array}$ & $\begin{array}{l}\text { a. } 2 \\
\text { a. } 3\end{array}$ & $\begin{array}{r}100 \\
2000\end{array}$ & & & like id 2221 & \\
\hline 8 & $2225 \cdots 2227$ & a.1 & 0000 & $1^{2}$ & $\begin{array}{l}3^{2} \\
{\left[1^{2}\right.}\end{array}$ & $\begin{array}{l}3^{2},(42)^{3} \\
3^{2},\left(1^{2}\right)^{3}\end{array}$ & $\begin{array}{c}(32)^{4},(41)^{9} \\
32]^{3}\end{array}$ \\
\hline
\end{tabular}

central quotient $G / \gamma_{c}(G)$, where $c=\operatorname{cl}(G)$ denotes the nilpotency class of $G$, and either $|G|=3|H|$, that is, $\gamma_{c}(G)$ is cyclic of order 3, or $|G|=9|H|$, that is, $\gamma_{c}(G)$ is bicyclic of type $(3,3)$. See also [[29], \$2.2, p. 410-411] and [[30], $\$ 4$, p. 163-164].

The vertices of the tree diagrams in Figure 1 and Figure 2 are classified by using various symbols:

1) big full discs $\bullet$ represent metabelian groups $\mathfrak{M}$ with defect $k(\mathfrak{M})=0$,

2) small full discs $\bullet$ represent metabelian groups $\mathfrak{M}$ with defect $k(\mathfrak{M})=1$.

In the Figures 3-5,

1) big full discs - represent metabelian groups $\mathfrak{M}$ with bicyclic centre of type $(3,3)$ and defect $k(\mathfrak{M})=0 \quad$ [[29], \$3.3.2, p. 429],

2) small full discs - represent metabelian groups $\mathfrak{M}$ with cyclic centre of order 3 and defect $k(\mathfrak{M})=1$,

3) small contour squares $\square$ represent non-metabelian groups $\mathfrak{G}$.

In the Figure 6 and Figure 7,

1) big contour squares $\square$ represent groups $\mathfrak{G}$ with relation rank $d_{2}(\mathfrak{G}) \leq 3$,

2) small contour squares $\square$ represent groups $\mathfrak{G}$ with relation rank $d_{2}(\mathfrak{G}) \geq 4$.

A symbol $n *$ adjacent to a vertex denotes the multiplicity of a batch of $n$ siblings, that is, immediate descendants sharing a common parent. The groups of particular importance are labelled by a number in angles, which is the identifier in the SmallGroups Library [25] [26] of GAP [32] and MAGMA [17]. We omit the orders, which are given on the left hand scale. The IPOD $\varkappa_{1}$ [[18], Thm. 2.5, Tbl. 6-7], in the bottom rectangle concerns all vertices located vertically above. The first, resp. second, component $\tau_{1}(1)$, resp. $\tau_{1}(2)$, of the IPAD [[1], Dfn. 3.3, p. 288] in the left rectangle concerns vertices $G$ on the same horizontal level with defect $k(G)=0$. The periodicity with length 2 of branches, $\mathcal{B}(j) \simeq \mathcal{B}(j+2)$ for $j \geq 4$, resp. $j \geq 7$, sets in with branch $\mathcal{B}(4)$, resp. $\mathcal{B}(7)$, having a root of order $3^{4}$, resp. $3^{7}$, in Figure 1 and Figure 2, resp. 3 and 4 . The metabelian skeletons of the Figure 3 and Figure 4 were drawn by 
Table 3. IPOD $\varkappa_{1} G$ and iterated IPAD $\tau_{*}^{(2)} G$ of 3-groups $G$ on $\mathcal{T}^{2}\left\langle 3^{5}, 6\right\rangle$.

\begin{tabular}{|c|c|c|c|c|c|c|c|}
\hline lo & id & type & $\varkappa_{1} G$ & $\tau_{0} G$ & $\tau_{0} H$ & $\tau_{1} H$ & $\tau_{2} H$ \\
\hline \multirow[b]{2}{*}{5} & \multirow[b]{2}{*}{6} & \multirow[b]{2}{*}{ c. 18} & \multirow[b]{2}{*}{0122} & \multirow[b]{2}{*}{$1^{2}$} & $1^{3}$ & $\left(1^{3}\right)^{4},\left(1^{2}\right)^{9}$ & $\left(1^{2}\right)^{13}$ \\
\hline & & & & & 21 & $1^{3},(21)^{3}$ & $\left.\left(1^{2}\right)^{4} \quad\right]^{3}$ \\
\hline \multirow{3}{*}{6} & \multirow{3}{*}{48} & \multirow{3}{*}{ H. 4} & \multirow{3}{*}{2122} & \multirow{3}{*}{$1^{2}$} & $2^{2}$ & $\left(21^{2}\right)^{4}$ & $1^{3},(21)^{12}$ \\
\hline & & & & & $1^{3}$ & $21^{2},\left(1^{3}\right)^{3},\left(1^{2}\right)^{9}$ & $1^{3},(21)^{3},\left(1^{2}\right)^{9}$ \\
\hline & & & & & 21 & $21^{2},(21)^{3}$ & $\left.1^{3},(21)^{3} \quad\right]^{2}$ \\
\hline 6 & $Q=49$ & c. 18 & 0122 & \multirow{6}{*}{$1^{2}$} & & IPAD like id 48 & \\
\hline 6 & 50 & E.14 & 3122 & & & IPAD like id 48 & \\
\hline 6 & 51 & E.6 & 1122 & & & IPAD like id 48 & \\
\hline \multirow{3}{*}{7} & \multirow{3}{*}{284} & \multirow{3}{*}{ c. 18} & \multirow{3}{*}{0122} & & $2^{2}$ & $\left(21^{2}\right)^{4}$ & $21^{2},\left(2^{2}\right)^{12}$ \\
\hline & & & & & $1^{3}$ & $\left(21^{2}\right)^{4},\left(1^{2}\right)^{9}$ & $21^{2},\left(1^{3}\right)^{3},(21)^{9}$ \\
\hline & & & & & 21 & $21^{2},(21)^{3}$ & $\left.21^{2},(21)^{3} \quad\right]^{2}$ \\
\hline \multirow{3}{*}{7} & \multirow{3}{*}{285} & \multirow{3}{*}{ c. 18} & \multirow{3}{*}{0122} & \multirow{3}{*}{$1^{2}$} & 32 & $2^{2} 1,\left(31^{2}\right)^{3}$ & $\left(21^{2}\right)^{4},(31)^{9}$ \\
\hline & & & & & $1^{3}$ & $2^{2} 1,\left(\mathbf{1}^{3}\right)^{3},\left(1^{2}\right)^{9}$ & $21^{2},\left(2^{2}\right)^{3},\left(1^{2}\right)^{9}$ \\
\hline & & & & & 21 & $2^{2} 1,(21)^{3}$ & $\left.21^{2},(21)^{3} \quad\right]^{2}$ \\
\hline 7 & $286 / 287$ & H.4 & 2122 & & & IPAD like id 285 & \\
\hline 7 & 288 & E.6 & 1122 & & & IPAD like id 285 & \\
\hline 7 & $289 / 290$ & E.14 & 3122 & & & IPAD like id 285 & \\
\hline \multirow{4}{*}{7} & \multirow{4}{*}{291} & \multirow{4}{*}{ c. 18} & \multirow{4}{*}{0122} & \multirow{4}{*}{$1^{2}$} & $2^{2}$ & $\left(21^{2}\right)^{4}$ & $21^{2},\left(2^{2}\right)^{3},(31)^{9}$ \\
\hline & & & & & $1^{3}$ & $21^{2},\left(1^{3}\right)^{3},\left(1^{2}\right)^{9}$ & $21^{2},(12)^{3},\left(1^{2}\right)^{9}$ \\
\hline & & & & & 21 & $21^{2},(31)^{3}$ & $21^{2},(21)^{3}$ \\
\hline & & & & & 21 & $21^{2},(21)^{3}$ & $21^{2},(21)^{3}$ \\
\hline \multirow{3}{*}{8} & \multirow{3}{*}{613} & & & & 32 & $2^{2} 1,\left(31^{2}\right)^{3}$ & $\left(2^{2} 1\right)^{4},(32)^{9}$ \\
\hline & & c. 18 & 0122 & $1^{2}$ & $1^{3}$ & $2^{2} 1,\left(21^{2}\right)^{3},\left(1^{2}\right)^{9}$ & $2^{2} 1,\left(1^{3}\right)^{3},\left(2^{2}\right)^{3},(21)^{6}$ \\
\hline & & & & & 21 & $2^{2} 1,(31)^{3}$ & $\left.2^{2} 1,\left(2^{2}\right)^{3} \quad\right]^{2}$ \\
\hline 8 & $614 / 615$ & H.4 & 2122 & & & IPAD like id 613 & \\
\hline 8 & 616 & E. 6 & 1122 & & & IPAD like id 613 & \\
\hline 8 & $617 / 618$ & E.14 & 3122 & & & IPAD like id 613 & \\
\hline
\end{tabular}

Nebelung [[37], p. $189 \mathrm{ff}]$, the complete trees were given by Ascione and coworkers [38], [[39], Fig. 4.8, p. 76, and Fig. 6.1, p. 123].

We define two kinds of arithmetically structured graphs $\mathcal{G}$ of finite $p$ groups by mapping each vertex $V \in \mathcal{G}$ of the graph to statistical number theoretic information, e.g. the distribution of second $p$-class groups $\mathfrak{M}=G_{p}^{2} K$ or $p$-class tower groups $G=\mathrm{G}_{p}^{\infty} K$, with respect to a given kind of number fields $K$, for instance real quadratic fields $K=K(d):=\mathbb{Q}(\sqrt{d})$ with discriminant 
Table 4. IPOD $\varkappa_{1} G$ and iterated IPAD $\tau_{*}^{(2)} G$ of 3-groups $G$ on $\mathcal{T}^{2}\left\langle 3^{5}, 8\right\rangle$.

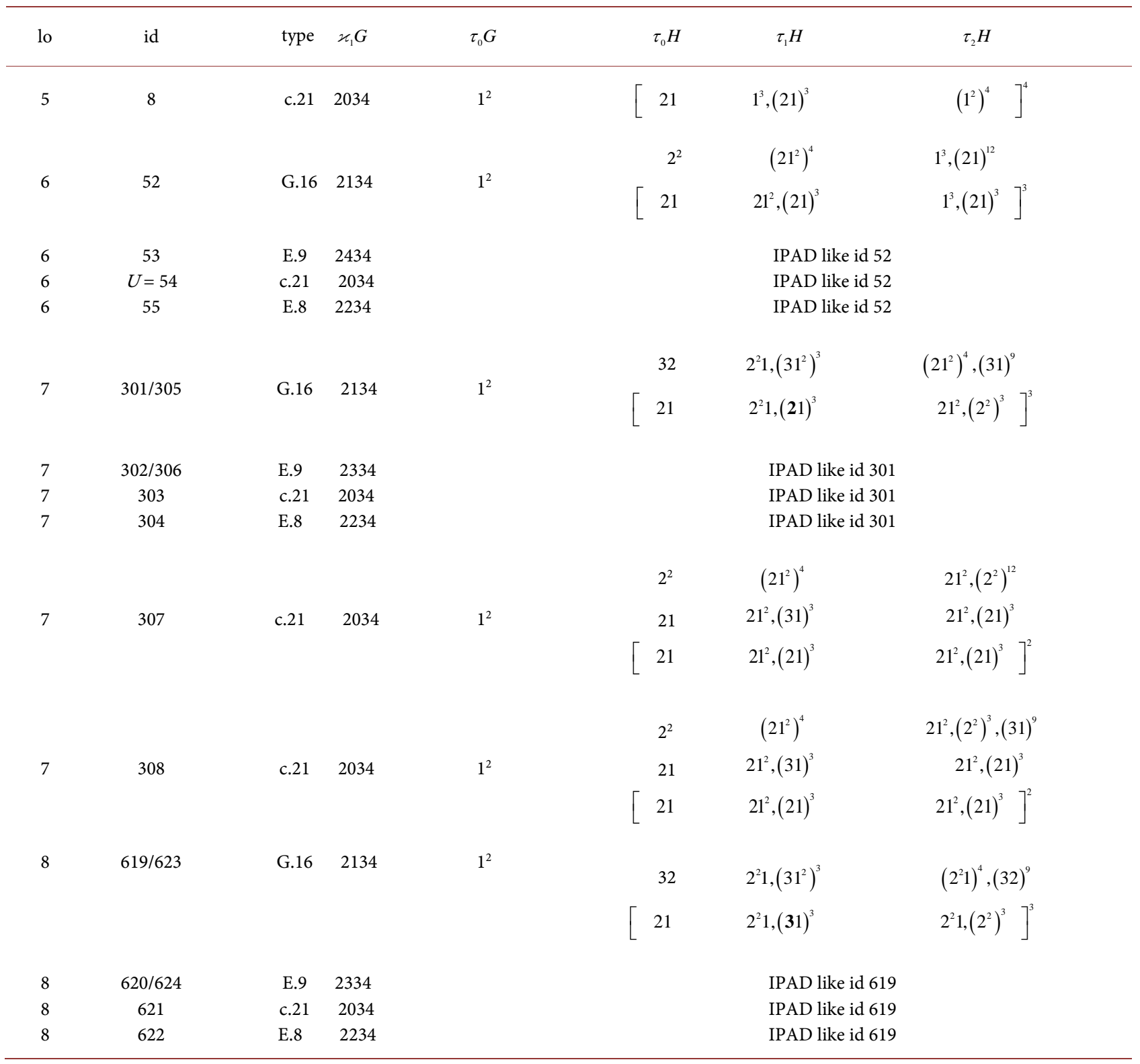

$d>0$.

Definition 6.2. Let $p$ be a prime and $\mathcal{G}$ be a subgraph of a descendant tree $\mathcal{T}$ of finite $p$-groups.

- The mapping

$$
\operatorname{MD}: \mathcal{G} \rightarrow \mathbb{N} \cup\{\infty\}, \quad V \mapsto \inf \left\{d \mid \mathrm{G}_{p}^{2} K(d) \simeq V\right\}
$$

is called the distribution of minimal discriminants on $\mathcal{G}$.

- For an assigned upper bound $B>0$, the mapping

$$
\mathrm{AF}: \mathcal{G} \rightarrow \mathbb{N} \cup\{0\}, \quad V \mapsto \#\left\{d<B \mid \mathrm{G}_{p}^{2} K(d) \simeq V\right\}
$$

is called the distribution of absolute frequencies on $\mathcal{G}$.

For both mappings, the subset of the graph $\mathcal{G}$ consisting of vertices $V$ with 
Table 5. IPOD $\varkappa_{1} G$ and iterated IPAD $\tau_{*}^{(2)} G$ of sporadic 3-groups $G$ of type H.4.

\begin{tabular}{|c|c|c|c|c|c|c|c|}
\hline lo & id & type & $\varkappa_{1} G$ & $\tau_{0} G$ & $\tau_{0} H$ & $\tau_{1} H$ & $\tau_{2} H$ \\
\hline \multirow{3}{*}{5} & \multirow{3}{*}{4} & \multirow{3}{*}{ H.4 } & \multirow{3}{*}{4111} & \multirow{3}{*}{$1^{2}$} & $1^{3}$ & $\left(1^{3}\right)^{4},\left(1^{2}\right)^{9}$ & $\left(1^{2}\right)^{13}$ \\
\hline & & & & & $1^{3}$ & $1^{3},(21)^{3},\left(1^{2}\right)^{9}$ & $\left(1^{2}\right)^{4},(2)^{9}$ \\
\hline & & & & & 21 & $1^{3},(21)^{3}$ & $\left(1^{2}\right)^{4}$ \\
\hline \multirow{3}{*}{6} & \multirow{3}{*}{$N=45$} & \multirow{3}{*}{ H.4 } & \multirow{3}{*}{4111} & \multirow{3}{*}{$1^{2}$} & $1^{3}$ & $21^{2},\left(1^{3}\right)^{3},\left(1^{2}\right)^{9}$ & $1^{3},(21)^{3},\left(1^{2}\right)^{9}$ \\
\hline & & & & & $1^{3}$ & $21^{2},(21)^{12}$ & $\left.21^{2},(21)^{12}\right]^{2}$ \\
\hline & & & & & 21 & $21^{2},(21)^{3}$ & $21^{2},\left(2^{2}\right)^{3}$ \\
\hline \multirow{3}{*}{7} & \multirow{3}{*}{270} & \multirow{3}{*}{ H.4 } & \multirow{3}{*}{4111} & \multirow{3}{*}{$1^{2}$} & $1^{3}$ & $\left(21^{2}\right)^{4},\left(1^{2}\right)^{9}$ & $21^{2},\left(1^{3}\right)^{3},(21)^{9}$ \\
\hline & & & & & $1^{3}$ & $21^{2},(21)^{12}$ & $\left.21^{2},(21)^{12}\right]^{2}$ \\
\hline & & & & & 21 & $21^{2},(21)^{3}$ & $21^{2},\left(2^{2}\right)^{3}$ \\
\hline \multirow{3}{*}{7} & \multirow{3}{*}{$271 / 272$} & \multirow{3}{*}{ H.4 } & \multirow{3}{*}{4111} & \multirow{3}{*}{$1^{2}$} & $1^{3}$ & $21^{2},\left(1^{3}\right)^{3},\left(1^{2}\right)^{9}$ & $21^{2},\left(2^{2}\right)^{3},\left(1^{2}\right)^{9}$ \\
\hline & & & & & $1^{3}$ & $21^{2},(21)^{12}$ & $\left.21^{2},(21)^{12}\right]^{2}$ \\
\hline & & & & & 21 & $21^{2},(31)^{3}$ & $21^{2},(21)^{3}$ \\
\hline \multirow{4}{*}{7} & \multirow{4}{*}{273} & \multirow{4}{*}{ H.4 } & \multirow{4}{*}{4111} & \multirow{4}{*}{$1^{2}$} & $1^{3}$ & $21^{2},\left(1^{3}\right)^{3},\left(1^{2}\right)^{9}$ & $21^{2},(21)^{3},\left(1^{2}\right)^{9}$ \\
\hline & & & & & $1^{3}$ & $21^{2},(21)^{12}$ & $21^{2},(21)^{12}$ \\
\hline & & & & & $1^{3}$ & $\left(21^{2}\right)^{4},\left(2^{2}\right)^{9}$ & $\left(21^{2}\right)^{13}$ \\
\hline & & & & & 21 & $21^{2},(21)^{3}$ & $21^{2},(21)^{3}$ \\
\hline \multirow{3}{*}{8} & \multirow{3}{*}{$605 / 606$} & \multirow{3}{*}{ H.4 } & \multirow{3}{*}{4111} & \multirow{3}{*}{$1^{2}$} & $1^{3}$ & $\left(21^{2}\right)^{4},\left(1^{2}\right)^{9}$ & $2^{2} 1,\left(1^{3}\right)^{3},\left(2^{2}\right)^{3},(21)^{6}$ \\
\hline & & & & & $1^{3}$ & $\left(21^{2}\right)^{4},\left(2^{2}\right)^{9}$ & $\left.2^{2} 1,\left(21^{2}\right)^{12}\right]^{2}$ \\
\hline & & & & & 21 & $21^{2},(31)^{3}$ & $2^{2} 1,\left(2^{2}\right)^{3}$ \\
\hline
\end{tabular}

$\operatorname{MD}(V) \neq \infty$, resp. $\operatorname{AF}(V) \neq 0$, is called the support of the distribution. The trivial values outside of the support will be ignored in the sequel.

Whereas Figure 1 displays an AF-distribution, the Figures 2-4 show MD distributions. The Figures 5-7 contain both distributions simultaneously.

\section{3-Class Towers of Quadratic Fields and Iterated IPADs of Second Order}

\subsection{3-Groups $G$ of Coclass cc $(G)=1$}

Table 2 shows the designation of the transfer kernel type [37], the IPOD $\varkappa_{1} G$, and the iterated multi-layered IPAD of second order,

$$
\tau_{*}^{(2)} G=\left[\tau_{0} G ;\left[\tau_{0} H ; \tau_{1} H ; \tau_{2} H_{H \in \mathrm{Ly} \mathrm{y}_{1} G}\right]\right],
$$

for 3-groups $G$ of maximal class up to order $|G|=3^{8}$, characterized by the logarithmic order, lo , i.e. $\operatorname{lo}(G):=\log _{3}|G|$, and the SmallGroup identifier, id. [25] [26]. 
Table 6. IPOD $\varkappa_{1} G$ and iterated IPAD $\tau_{*}^{(2)} G$ of sporadic 3-groups $G$ of type G.19.

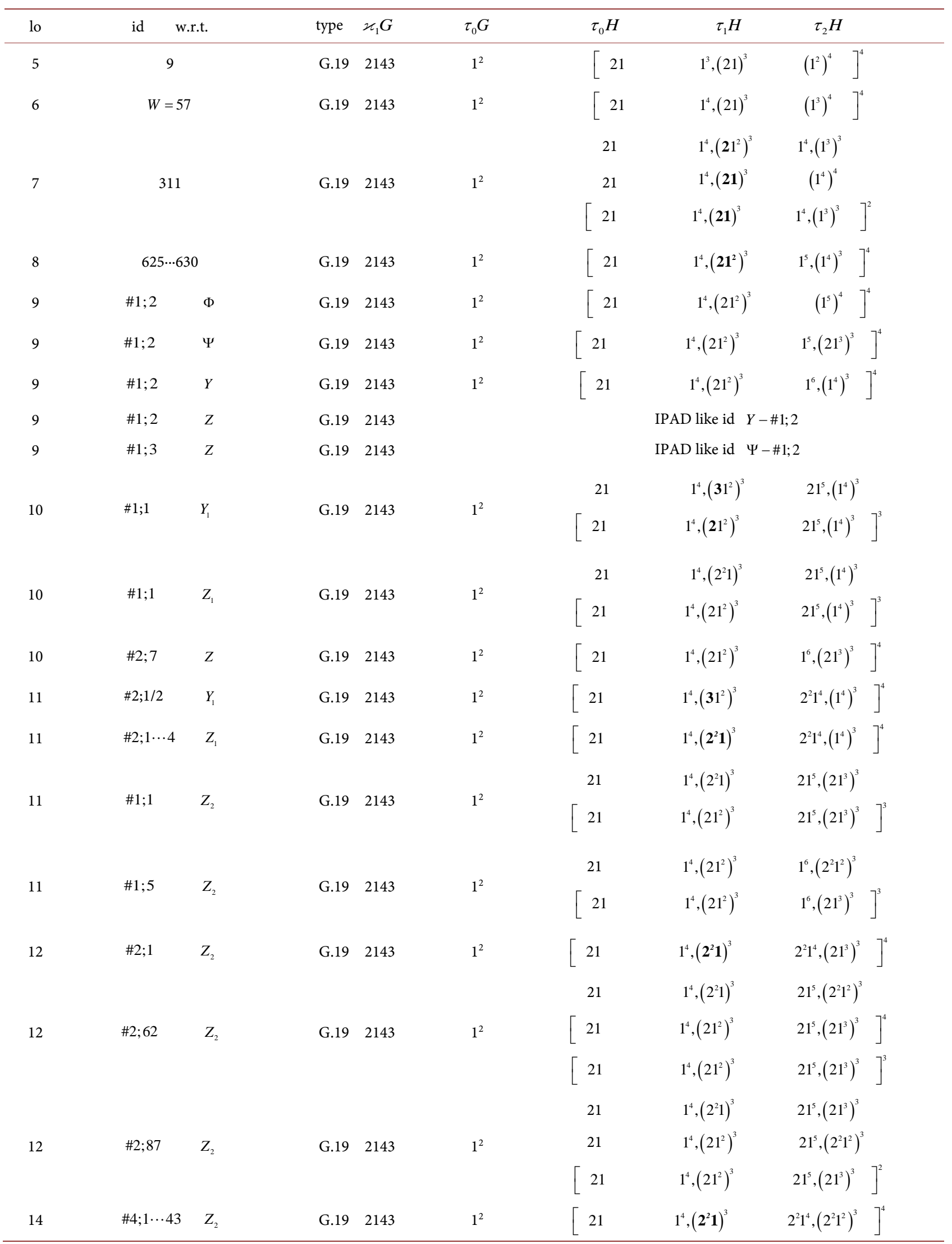




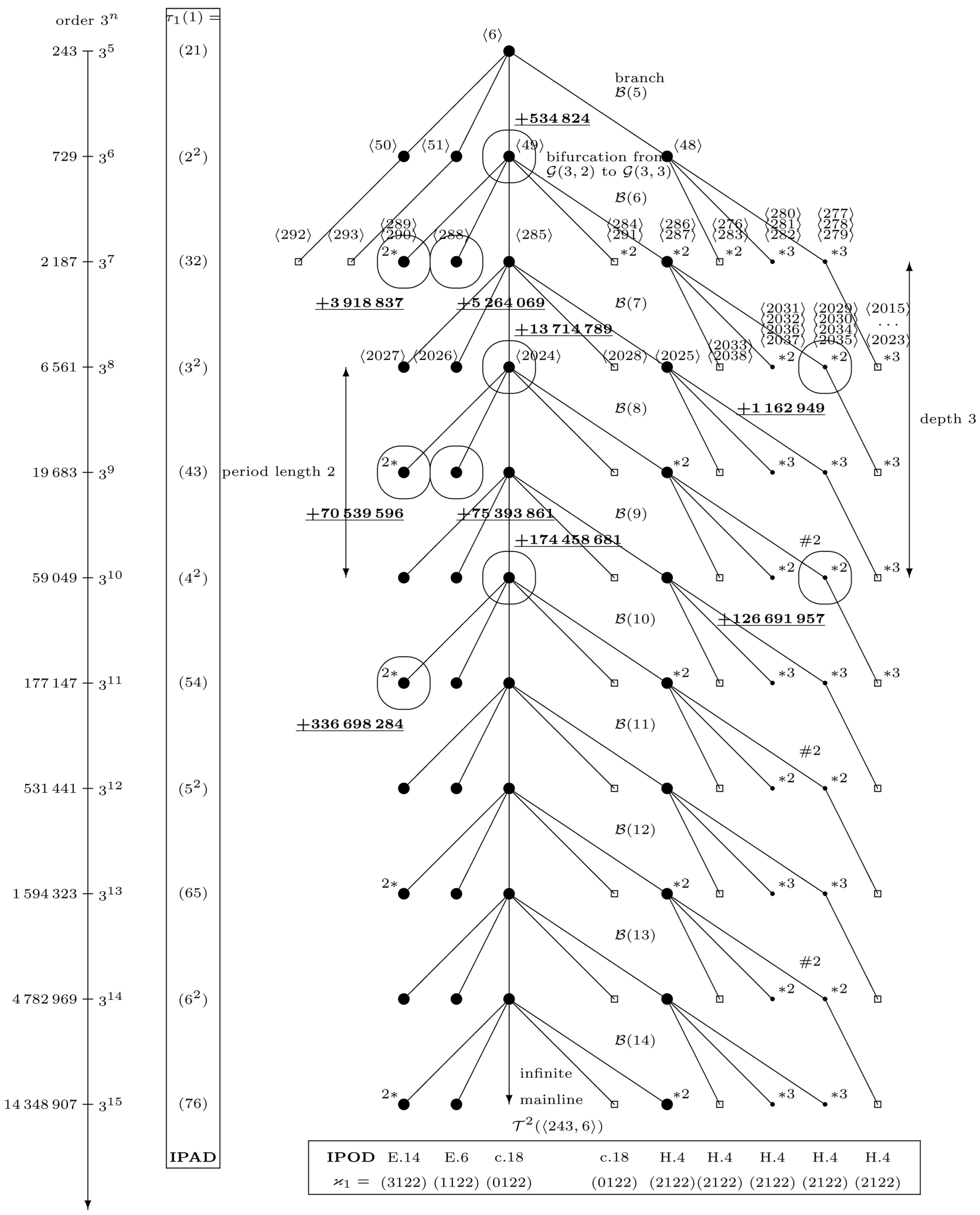

Figure 3. Distribution of minimal discriminants for $G_{3}^{2} K$ on the coclass tree $\mathcal{T}^{2}\langle 243,6\rangle$.

The groups in Table 2 are represented by vertices of the tree diagrams in Figure 1 and Figure 2. 


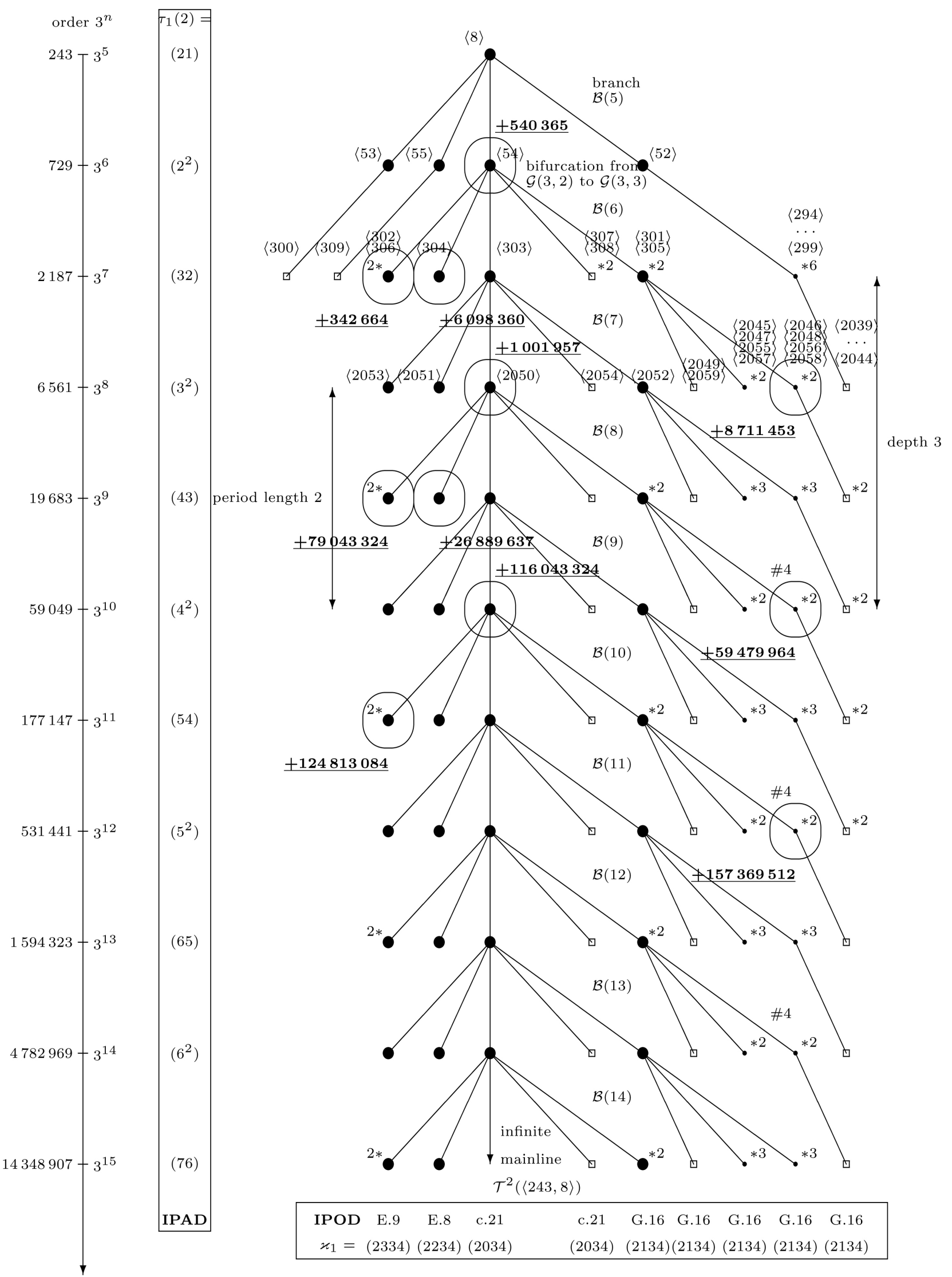

Figure 4. Distribution of minimal discriminants for $G_{3}^{2} K$ on the coclass tree $\mathcal{T}^{2}\langle 243,8\rangle$. 


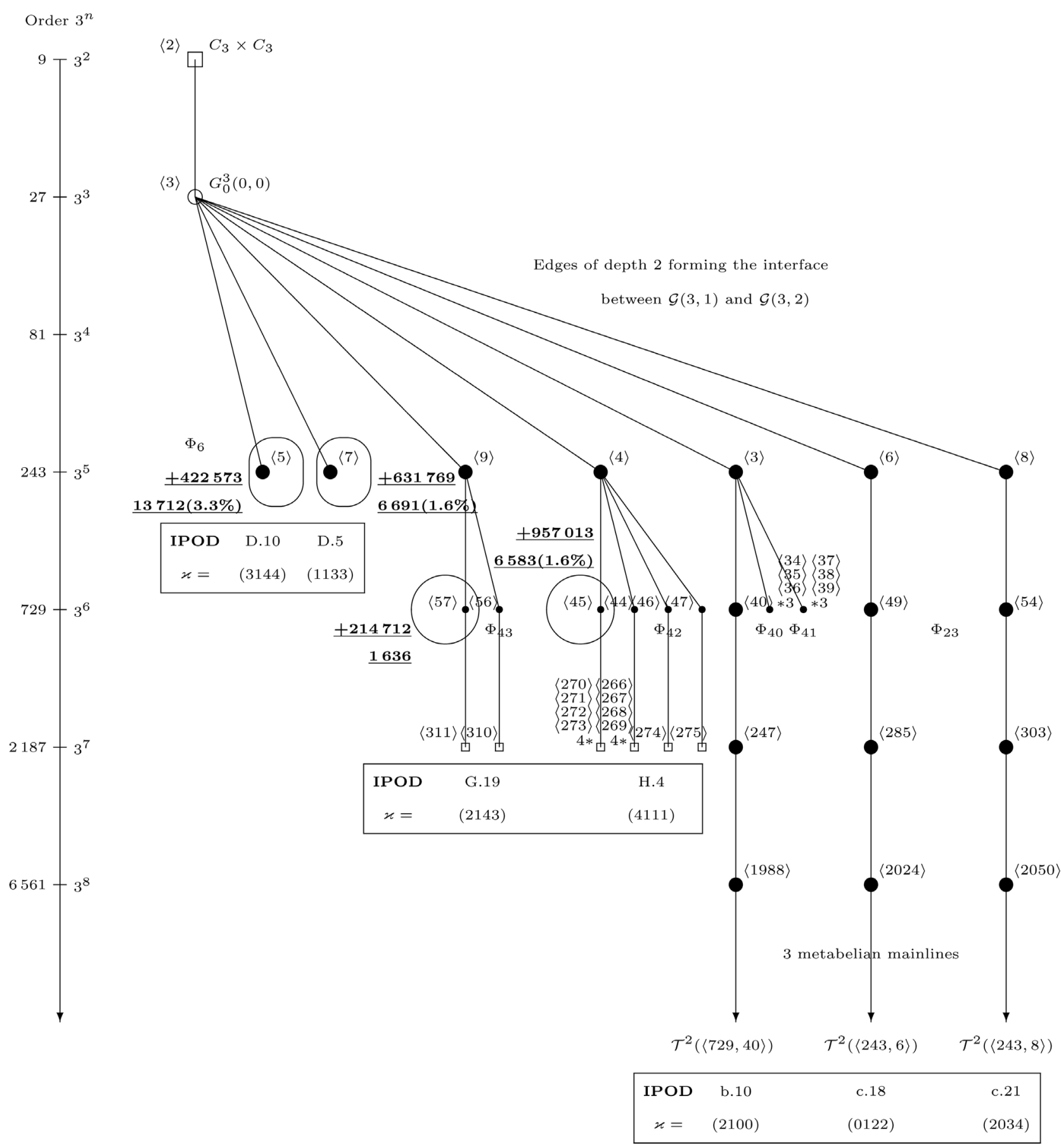

Figure 5. Distribution of second 3-class groups $G_{3}^{2} K$ on the sporadic graph $\mathcal{G}_{0}(3,2)$.

\subsection{Real Quadratic Fields of Types a.1, a.2 and a.3}

Sound numerical investigations of real quadratic fields $K=\mathbb{Q}(\sqrt{d})$ with fundamental discriminant $d>0$ started in 1982, when Heider and Schmithals [40] showed the first examples of a Galois cohomology structure of Moser's type $\alpha$ on unit groups of unramified cyclic cubic extensions, $L / K$ which are dihedral of degree 6 over $\mathbb{Q}$ [[5], Prop. 4.2, p. 482], and of IPODs $\varkappa_{1} K$ with type a.1 $(d=62501)$, type a.2 $(d=72329)$, and type a.3 $(d=32009)$, in the 


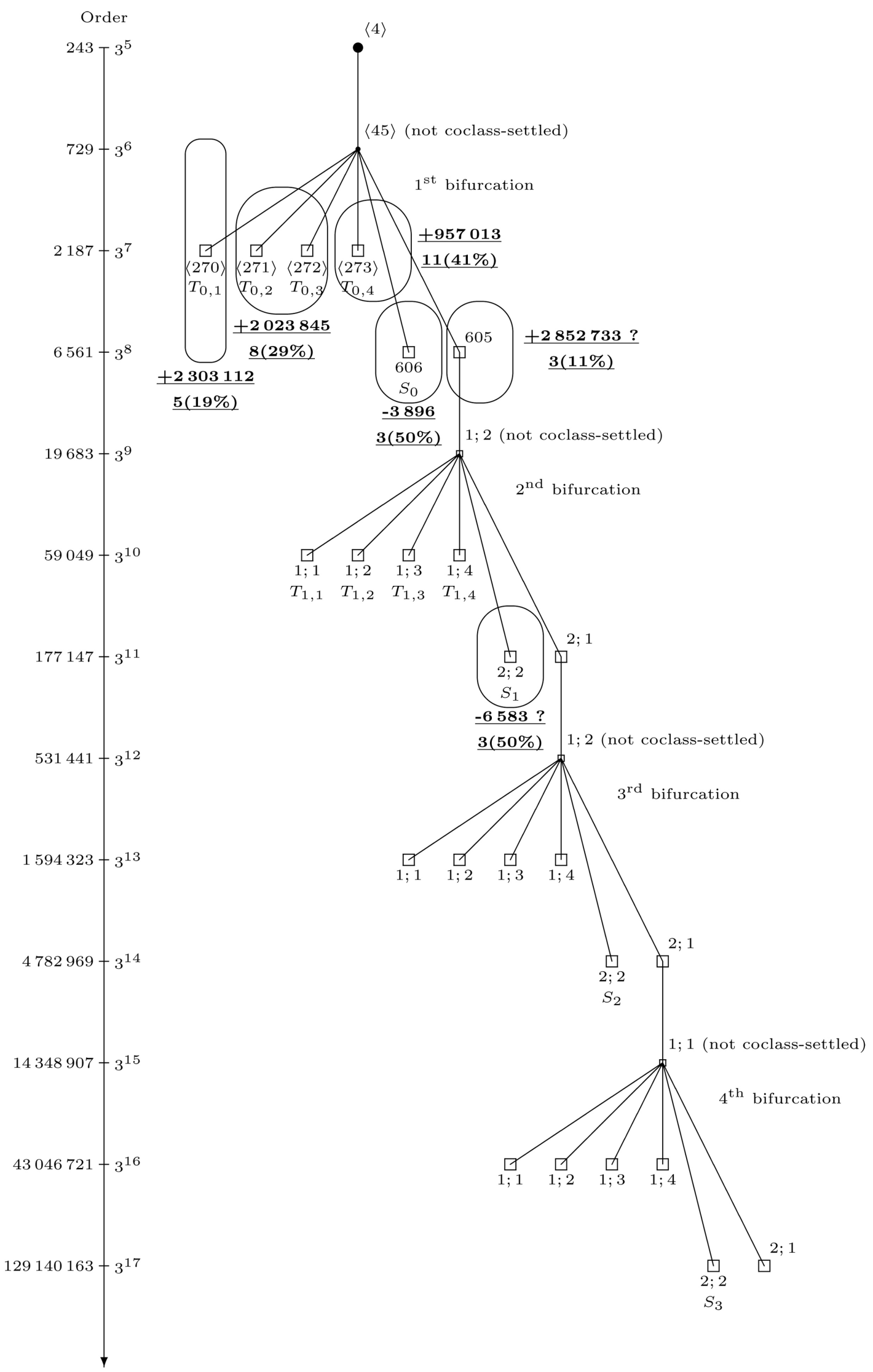

Figure 6. Distribution of 3-class tower groups $\mathrm{G}_{3}^{\infty} K$ on the descendant tree $\mathcal{T}_{*}\langle 243,4\rangle$. 


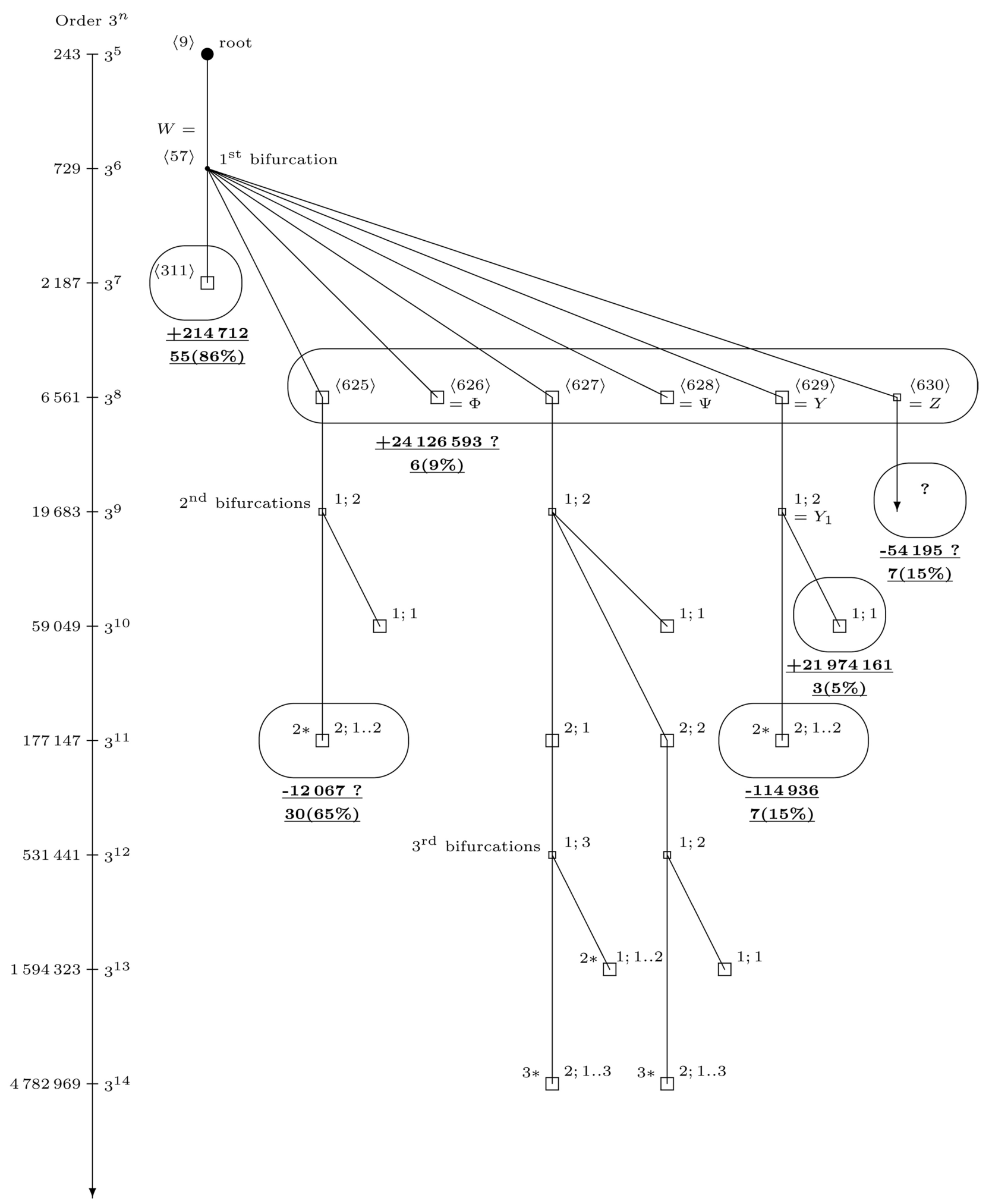

Figure 7. Distribution of 3-class tower groups $\mathrm{G}_{3}^{\infty} K$ on the descendant tree $\mathcal{T}_{*}\langle 243,9\rangle$.

notation of Nebelung [37]. See Figure 2.

Our extension in 1991 [41] merely produced further examples for these occurrences of type a. In the 15 years from 1991 to 2006 we consequently were 
convinced that this type with at least three total 3-principalizations is the only possible type of real quadratic fields.

The absolute frequencies in [[5], Tbl. 2, p. 496] and [[24], Tbl. 6.1, p. 451], which should be corrected by the Corrigenda in the Appendix, and the extended statistics in Figure 1 underpin the striking dominance of type a. The distribution of the second 3-class groups $\mathfrak{M}=\mathrm{G}_{3}^{2} K$ with the smallest order $3^{4}$, resp. $3^{6}$, alone reaches $79.7 \%$ for the accumulated types a.2 and a. 3 together, resp. $6.4 \%$ for type a.1.

So it is not astonishing that the first exception $d=214712$ without any total 3-principalizations did not show up earlier than in 2006 [[5], Tbl. 4, p. 498], [[24], Tbl. 6.3, p. 452]. See Figure 5.

The most extensive computation of data concerning unramified cyclic cubic extensions $L / K$ of the 481,756 real quadratic fields $K=\mathbb{Q}(\sqrt{d})$ with discriminant $0<d<10^{9}$ and 3-class rank $\rho_{3} K=2$ has been achieved by M. R. Bush in 2015 [42]. In the following, we focus on the partial results for 3-class groups of type (3,3), since they extend our own results of 2010 [5] [24].

Proposition 7.1. (IPADs of fields with type a up to $d<10^{9} \quad[42]$ )

In the range $0<d<10^{9}$ with 415,698 fundamental discriminants $d$ of real quadratic fields $K=\mathbb{Q}(\sqrt{d})$ having 3-class group of type $(3,3)$, there exist precisely

208236 cases $(50.1 \%)$ with IPAD $\tau^{(1)} K=\left[1^{2} ; 21,\left(1^{2}\right)^{3}\right]$,

122955 cases (29.6\% ) with IPAD $\tau^{(1)} K=\left[1^{2} ; 1^{3},\left(1^{2}\right)^{3}\right]$,

26678 cases $(6.4 \%)$ with IPAD $\tau^{(1)} K=\left[1^{2} ; 2^{2},\left(1^{2}\right)^{3}\right]$, and

11780 cases ( $2.8 \%$ ) with IPAD $\tau^{(1)} K=1\left[{ }^{2} ; 32,\left(1^{2}\right)^{3}\right]$.

Proof. The results were computed with PARI/GP [14], double-checked with MAGMA [17], and kindly communicated to us by M. R. Bush, privately [42].

For establishing the connection between IPADs and IPODs we need the following bridge.

Corollary 7.1. (Associated IPODs of fields with type a)

1) A real quadratic field $K$ with IPAD $\tau^{(1)} K=\left[1^{2} ; 21,\left(1^{2}\right)^{3}\right]$ has IPOD either $\varkappa_{1} K=(1000)$ of type a.2 or $\varkappa_{1} K=(2000)$ of type a.3.

2) A real quadratic field $K$ with IPAD $\tau^{(1)} K=\left[1^{2} ; 1^{3},\left(1^{2}\right)^{3}\right]$ has IPOD $\varkappa_{1} K=(2000)$ of type a.3, more precisely a. $3^{*}$, in view of the exceptional IPAD.

3) A real quadratic field $K$ with IPAD $\tau^{(1)} K=\left[1^{2} ; 2^{2},\left(1^{2}\right)^{3}\right]$ has IPOD $\varkappa_{1} K=(0000)$ of type a.1.

4) A real quadratic field $K$ with IPAD $\tau^{(1)} K=\left[1^{2} ; 32,\left(1^{2}\right)^{3}\right]$ has IPOD either $\varkappa_{1} K=(1000)$ of type $a .2$ or $\varkappa_{1} K=(2000)$ of type a.3.

Proof. Here, we again make use of the selection rule [[29], Thm. 3.5, p. 420] that only every other branch of the tree $\mathcal{T}^{1}\left\langle 3^{2}, 2\right\rangle$ is admissible for second 3-class groups $\mathfrak{M}=G_{3}^{2} K$ of (real) quadratic fields $K$.

According to Table 2, three (isomorphism classes of) groups $G$ share the 
common IPAD $\tau^{(1)} G=\left[1^{2} ; 21,\left(1^{2}\right)^{3}\right]$, namely $\langle 81,8 \cdots 10\rangle$, whereas the IPAD $\tau^{(1)} G=\left[1^{2} ; 1^{3},\left(1^{2}\right)^{3}\right]$ unambiguously leads to the group $\langle 81,7\rangle$ with IPOD $\varkappa_{1} G=(2000)$.

In Theorem 7.4 we shall show that the mainline group $\langle 81,9\rangle$ cannot occur as the second 3-class group of a real quadratic field. Among the remaining two possible groups, $\langle 81,8\rangle$ has IPOD $\varkappa_{1} G=(2000)$ and $\langle 81,10\rangle$ has IPOD $\varkappa_{1} G=(1000)$.

The IPAD $\tau^{(1)} G=\left[1^{2} ; 2^{2},\left(1^{2}\right)^{3}\right]$ leads to three groups $\langle 729,99 \cdots 101\rangle$ with IPOD $\varkappa_{1} G=(0000)$ and defect of commutativity $k=1$ [[29], \$3.1.1, p. 412]. Concerning the IPAD $\tau^{(1)} G=\left[1^{2} ; 32,\left(1^{2}\right)^{3}\right]$, Table 2 yields four groups $G$ with SmallGroup identifiers $\langle 729,95 \cdots 98\rangle$. The mainline group $\langle 729,95\rangle$ is discouraged by Theorem 7.4, $\langle 729,96\rangle$ has IPOD $\varkappa_{1} G=(1000)$, and the two groups $\langle 729,97 \cdots 98\rangle$ have IPOD $\varkappa_{1} G=(2000)$.

By the Artin reciprocity law [9] [11], the Artin pattern $\mathrm{AP}(K)$ of the field $K$ coincides with the Artin pattern $\mathrm{AP}(\mathfrak{M})$ of its second 3-class group $\mathfrak{M}=\mathrm{G}_{3}^{2} K$.

Remark 7.1. The huge statistical ensembles underlying the computations of Bush [42] admit a prediction of sound and reliable tendencies in the population of the ground state. If we compare the smaller range $d<10^{7}$ in [5] with the extended range $d<10^{9}$ in [42], then we have a decrease

$$
\frac{1382}{2576} \approx 53.6 \% \searrow \frac{208236}{415698} \approx 50.1 \% \text { by } 3.5 \% \text { for the union of types a.2 and }
$$

a.3,

and increases

$\frac{698}{2576} \approx 27.1 \% \nearrow \frac{122955}{415698} \approx 29.6 \%$ by $2.5 \%$ for type $a .3^{*}$, and

$\frac{150}{2576} \approx 5.8 \% \nearrow \frac{26678}{415698} \approx 6.4 \%$ by $0.6 \%$ for type a. 1 .

Of course, the accumulation of all types a.2, a.3, and a. $3^{*}$ with absolute frequencies

$1382+698=2080$, resp. $208236+122955=331191$, shows a resultant decrease

$$
\frac{2080}{2576} \approx 80.7 \% \searrow \frac{331191}{415698} \approx 79.7 \% \text { by } 1.0 \% \text {. }
$$

For the union of the first excited states of types a.2 and a.3, we have a stagnation

$$
\frac{72}{2576} \approx 2.8 \% \approx \frac{11780}{415698} \approx 2.8 \% \text { at the same percentage. }
$$

Unfortunately, the exact absolute frequency of the ground state of type a.2, resp. type a.3, is unknown for the extended range $d<10^{9}$. It could be computed using Theorem 7.1. However, meanwhile we succeeded in separating all states of type a.2 and type a.3 up to $d<10^{8}$ by immediately figuring out the 3 - 
principalization type with MAGMA V2.22-1. In [21], we compare the results of this most recent tour de force of computing with asymptotic densities predicted by Boston, Bush and Hajir (communicated privately and yet unpublished, similar to [13]).

Figure 1 visualizes 3-groups of section $\$ 7.1$ which arise as 3-class tower groups $G=G_{3}^{\infty} K$ of real quadratic fields $K=\mathbb{Q}(\sqrt{d}), d>0$, with principalization types a.2 and a.3 and the corresponding absolute frequencies and percentages (relative frequencies with respect to the total number of 415,698 real quadratic fields with discriminants in the range $0<d<B$ for $B=10^{9}$ ) which were given in Proposition 7.1.

Figure 2 visualizes 3-groups of section $\$ 7.1$ which arise as 3-class tower groups $G=G_{3}^{\infty} K$ of real quadratic fields $K=\mathbb{Q}(\sqrt{d}), d>0$, with 3principalization types a.1, a.2 and a.3 and the corresponding minimal discriminants in the sense of Definition 6.2.

As mentioned in [1], we have the following criterion for distinguishing subtypes of type a:

Theorem 7.1. (The ground state of type a [[29], \$3.2.5, pp. 423-424])

The second 3-class groups $\mathfrak{M}=\mathrm{G}_{3}^{2} \mathrm{~K}$ with the smallest order $3^{4}$ possessing type $a .2$ or a.3 can be separated by means of the iterated IPAD of second order $\tau^{(2)} \mathfrak{M}=\left[\tau_{0} \mathfrak{M} ;\left[\tau_{0} H ; \tau_{1} H\right]_{H \in \mathrm{Lyr}_{1} \mathfrak{M}}\right]$.

Proof. This is essentially [[1], Thm. 6.1, p. 296] but can also be seen directly by comparing the column $\tau_{1} H$ with the IPAD for the rows with lo $=4$ and id $\in\{7, \cdots, 10\}$ in Table 2 . Here the column $\tau_{2} H$, containing the second layer of the IPAD, does not permit a distinction.

Unfortunately, we also must state a negative result:

Theorem 7.2. (Excited states of type a [[29], \$3.2.5, pp. 423-424])

Even the multi-layered IPAD $\tau_{*}^{(2)} \mathfrak{M}=\left[\tau_{0} \mathfrak{M} ;\left[\tau_{0} H ; \tau_{1} H ; \tau_{2} H\right]_{H \in \mathrm{Lyr}_{1} \mathfrak{M}}\right]$ of second order is unable to separate the second 3-class groups $\mathfrak{M}=\mathrm{G}_{3}^{2} \mathrm{~K}$ with order $3^{6}$ and type a.2 or a.3. It is also unable to distinguish between the three candidates for $\mathfrak{M}$ of type a.1, and between the two candidates for $\mathfrak{M}$ of type a.3, both for orders $|\mathfrak{M}| \geq 3^{6}$.

Proof. This is a consequence of comparing both columns $\tau_{1} H$ and $\tau_{2} H$ for the rows with $l o \in\{6,8\}$ and id $\in\{95, \cdots, 101\}$, resp. id $\in\{2221, \cdots, 2227\}$ in Table 2. According to the selection rule [[29], Thm. 3.5, p. 420], only every other branch of the tree $\mathcal{T}^{1}\left\langle 3^{2}, 2\right\rangle$ is admissible for second 3-class groups $\mathfrak{M}=\mathrm{G}_{3}^{2} K$ of (real) quadratic fields $K$.

Theorem 7.3. (Two-stage 3-class towers of type a) For each (real) quadratic field $K$ with second 3 -class group $\mathfrak{M}=\mathrm{G}_{3}^{2} K$ of maximal class the 3-class tower has exact length $\ell_{3} K=2$.

Proof. Let $G$ be a 3-group of maximal class. Then $G$ is metabelian by [[29], Thm. 3.7, proof, p. 421] or directly by [[43], Thm. 6, p. 26]. Suppose that $H$ is a non-metabelian 3-group of derived length $\mathrm{dl}(H) \geq 3$ such that $H / H^{\prime \prime} \simeq G$. According to [[4], Thm. 5.4], the Artin patterns $\mathrm{AP}(H)$ and $\mathrm{AP}(G)$ coincide, 
in particular, both groups share a common IPOD $\varkappa_{1} H=\varkappa_{1} G$, which contains at least three total kernels, indicated by zeros, $\varkappa_{1}=(* 000)$ [18]. However, this is a contradiction already, since any non-metabelian 3-group, which necessarily must be of coclass at least 2 , is descendant of one of the five groups $\langle 243, n\rangle$ with $n \in\{3,4,6,8,9\}$ whose IPODs possess at most two total kernels, and a descendant cannot have an IPOD with more total kernels than its parent, by [[4], Thm. 5.2]. Consequently, the $\operatorname{cover} \operatorname{cov}(G)$ of $G$ in the sense of [[23], Dfn. 5.1] consists of the single element $G$.

Finally, we apply this result to class field theory: Since $\mathfrak{M}=\mathrm{G}_{3}^{2} K$ is assumed to be of coclass $\operatorname{cc}(\mathfrak{M})=1$, we obtain $G=G_{3}^{\infty} K \in \operatorname{cov}(\mathfrak{M})=\{\mathfrak{M}\}$ and the length of the 3 -class tower is given by $\ell_{3} K=\mathrm{dl}(G)=\mathrm{dl}(\mathfrak{M})=2$.

Remark 7.2. To the very best of our knowledge, Theorem 7.3 does not appear in the literature, although we are convinced that it is well known to experts, since it can also be proved purely group theoretically with the aid of a theorem by Blackburn [[43], Thm. 4, p. 26]. Here we prefer to give a new proof which uses the structure of descendant trees.

Theorem 7.4. (The forbidden mainline of coclass 1) The mainline vertices of the coclass- 1 tree cannot occur as second 3 -class groups $\mathfrak{M}=\mathrm{G}_{3}^{2} K$ of (real) quadratic fields $K$ (of type a.1).

Proof. Since periodicity sets in with branch $\mathcal{B}(4)$ in the Figure 1 and Figure 2 , and MAGMA shows that the groups $\left\langle 3^{3}, 3\right\rangle$ and $\left\langle 3^{4}, 9\right\rangle$ have $p$-multiplicator rank 4, all mainline vertices $V$ must have $p$-multiplicator rank $\mu(V)=4$ and thus relation rank $d_{2} V=\mu(V)=4$. However, a real quadratic field $K$ has torsion free Dirichlet unit rank $r=1$ and certainly does not contain the (complex) primitive third roots of unity. According to the corrected version [[23], Thm. 5.1] of the Shafarevich theorem [44], the relation rank $d_{2} G$ of the 3-tower group $G=G_{3}^{\infty} K$, which coincides with the second 3-class group $\mathfrak{M}=\mathrm{G}_{3}^{2} K$ by Theorem 7.3 , is bounded by $2=\rho \leq d_{2} G \leq \rho+r=2+1=3$, where $\rho=2$ denotes the 3-class rank of $K$.

\subsection{3-Groups $G$ of Coclass cc $(G)=2$ Arising from $\left\langle 3^{5}, 6\right\rangle$}

Table 3 shows the designation of the transfer kernel type [6] [37], the IPOD $\varkappa_{1} G$, and the iterated multi-layered IPAD of 2 nd order,

$$
\tau_{*}^{(2)} G=\left[\tau_{0} G ;\left[\tau_{0} H ; \tau_{1} H ; \tau_{2} H\right]_{H \in \mathrm{Lyr}_{1} G}\right],
$$

for 3-groups $G$ on the coclass tree $\mathcal{T}^{2}\left\langle 3^{5}, 6\right\rangle$ up to order $|G|=3^{8}$, characterized by the logarithmic order, lo , and the SmallGroup identifier, id , [25] [26]. To enable a brief reference for relative identifiers we put $Q:=\left\langle 3^{6}, 49\right\rangle$, since this group was called the non-CF group $Q$ by Ascione [38] [39].

The groups in Table 3 are represented by vertices of the tree diagram in Figure 3.

Theorem 7.5. (Smallest possible 3-tower groups $G=\mathrm{G}_{3}^{\infty} K$ of type E.6 or E.14 [1]). Let $G$ be a finite 3-group with IPAD of first order $\tau^{(1)} G=\left[\tau_{0} G ; \tau_{1} G\right]$, 
where $\tau_{0} G=1^{2}$ and $\tau_{1} G=\left(32,1^{3},(21)^{2}\right)$ is given in ordered form.

If the IPOD of $G$ is of type E.6, $\varkappa_{1} G=(1122)$, resp. E.14, $\varkappa_{1} G=(3122) \sim(4122)$, then the IPAD of second order $\tau^{(2)} G=\left[\tau_{0} G ;\left(\tau_{0} H_{i} ; \tau_{1} H_{i}\right)_{1 \leq i \leq 4}\right]$, where the maximal subgroups of index 3 in $G$ are denoted by $H_{1}, \cdots, H_{4}$, determines the isomorphism type of $G$ in the following way:

1) $\tau^{(1)} H_{2}=\left[1^{3} ; 2^{2} 1,\left(1^{3}\right)^{3},\left(1^{2}\right)^{9}\right]$ if and only if $\tau^{(1)} H_{i}=\left[21 ; 2^{2} 1,(21)^{3}\right]$ for $i \in\{3,4\}$ if and only if $G \simeq\left\langle 3^{7}, \mathbf{2 8 8}\right\rangle$, resp. $G \simeq\left\langle 3^{7}, \mathbf{2 8 9}\right\rangle$ or $G \simeq\left\langle 3^{7}, \mathbf{2 9 0}\right\rangle$, 2) $\tau^{(1)} H_{2}=\left[1^{3} ; 2^{2} 1,\left(21^{2}\right)^{3},\left(1^{2}\right)^{9}\right]$ if and only if $\tau^{(1)} H_{i}=\left[21 ; 2^{2} 1,(31)^{3}\right]$ for $i \in\{3,4\}$ if and only if $G \simeq\left\langle 3^{8}, 616\right\rangle$, resp. $G \simeq\left\langle 3^{8}, 617\right\rangle$ or $G \simeq\left\langle 3^{8}, 618\right\rangle$, whereas the component $\tau^{(1)} H_{1}=\left[32 ; 2^{2} 1,\left(31^{2}\right)^{3}\right]$ is fixed and does not admit a distinction.

Proof. This is essentially [[1], Thm. 6.2, pp. 297-298]. It is also an immediate consequence of Table 3, which has been computed with MAGMA [17]. As a termination criterion we can now use the more precise [[4], Thm. 5.1] instead of [[7], Cor. 3.0.1, p. 771].

Figure 3 visualizes 3-groups which arise as second 3-class groups $\mathfrak{M}=\mathrm{G}_{3}^{2} K$ of real quadratic fields $K=\mathbb{Q}(\sqrt{d}), d>0$, with 3-principalization types E.6 and E.14 in section $₫ 7.3$ and the corresponding minimal discriminants.

\subsection{Parametrized IPADs of Second Order for the Coclass Tree}

$$
\mathcal{T}^{2}\left\langle 3^{5}, 6\right\rangle
$$

Let $G \in \mathcal{T}^{2}\left\langle 3^{5}, 6\right\rangle$ be a descendant of coclass $\operatorname{cc}(G)=2$ of the root $\left\langle 3^{5}, 6\right\rangle$. Denote by $c:=\operatorname{cl}(G)$ the nilpotency class of $G$, by $t:=\mathrm{dl}(G)-2$ the indicator of a three-stage group, and by $k:=k(G)$, resp $k:=k(\pi G)$, the defect of commutativity of $G$ itself if $t=0$, and of the metabelian parent $\pi G$ if $t=1$.

Theorem 7.6. In dependence on the parameters $c, t$ and $k$, the IPAD of second order of $G$ has the form

$$
\begin{aligned}
\tau^{(2)} G=1^{2} ;(A(3, c- & \left.k-t) ; A(3, c-1-k-t) \times C_{3},\left(B(3, c-1-k-t) \times C_{3}\right)^{3}\right), \\
& \left(21 ; A(3, c-1-k-t) \times C_{3},(21)^{3}\right), \\
& \left(1^{3} ; A(3, c-1-k-t) \times C_{3},\left(1^{3}\right)^{3},\left(1^{2}\right)^{9}\right), \\
& \left(21 ; A(3, c-1-k-t) \times C_{3},(21)^{3}\right),
\end{aligned}
$$

where a variant of the nearly homocyclic abelian 3-group of order $n \geq 2$ in Definition 5.1, which can also be defined by $\mathrm{A}(3,0):=1, \mathrm{~A}(3,1):=C_{3}$, and

$$
\mathrm{A}(3, n):=\left\{\begin{array}{cccc}
C_{3^{m+1}} \times C_{3^{m}} & \text { if } & n=2 m+1 & \text { odd, } \\
C_{3^{m}} \times C_{3^{m}} & \text { if } & n=2 m & \text { even, }
\end{array}\right.
$$

is given by $\mathrm{B}(3,2):=C_{3^{2}}$ and 


$$
\mathrm{B}(3, n):=\left\{\begin{array}{llll}
C_{3^{m+1}} \times C_{3^{m}} & \text { if } & n=2 m+1 & \text { odd, } \\
C_{3^{m+2}} \times C_{3^{m}} & \text { if } & n=2 m+2 & \text { even. }
\end{array}\right.
$$

\subsection{Number Fields with IPOD of Type E.6 or E.14}

Let $K$ be a number field with 3-class group $\mathrm{Cl}_{3} K \simeq C_{3} \times C_{3}$ and first layer $\operatorname{Lyr}_{1} K=\left\{L_{1}, \cdots, L_{4}\right\}$ of unramified abelian extensions.

Theorem 7.7. (Criteria for $\ell_{3} K \in\{2,3\}$.) Let the IPOD of $K$ be of type E.6, $\varkappa_{1} K \sim(1313)$, resp. E.14, $\varkappa_{1} K \sim(2313)$. If $\tau_{1} K \sim\left(\mathrm{A}(3, c), 21,1^{3}, 21\right)$ with $c \geq 4$, then

$$
\begin{aligned}
\ell_{3} K=2 & \Leftrightarrow \tau_{1} L_{3} \sim\left(\mathrm{A}(3, c-1) \times C_{3},\left(\mathbf{1}^{3}\right)^{3},\left(1^{2}\right)^{9}\right) \\
& \Leftrightarrow \tau_{1} L_{j} \sim\left(\mathrm{A}(3, c-1) \times C_{3},(\mathbf{2 1})^{3}\right) \text { for } j \in\{2,4\}, \\
-\ell_{3} K=3 & \Leftrightarrow \tau_{1} L_{3} \sim\left(\mathrm{A}(3, c-1) \times C_{3},\left(\mathbf{2 1}^{2}\right)^{3},\left(1^{2}\right)^{9}\right) \\
& \Leftrightarrow \tau_{1} L_{j} \sim\left(\mathrm{A}(3, c-1) \times C_{3},(\mathbf{3 1})^{3}\right) \text { for } j \in\{2,4\} .
\end{aligned}
$$

Proof. Exemplarily, we conduct the proof for $c=5$, which is the most important situation for our computational applications.

Searching for the Artin pattern $\operatorname{AP}_{1}=\left(\tau_{1}, \varkappa_{1}\right)$ with $\tau_{1} \sim\left(32,21,1^{3}, 21\right)$ and $\varkappa_{1} \sim(1313)$, resp. (2313), in the descendant tree $\mathcal{T}(R)$ with abelian root $R:=\left\langle 3^{2}, 2\right\rangle \simeq C_{3} \times C_{3}$, unambiguously leads to the unique metabelian descendant with path $R \leftarrow\left\langle 3^{3}, 3\right\rangle \leftarrow\left\langle 3^{5}, 6\right\rangle \leftarrow\left\langle 3^{6}, 49\right\rangle \leftarrow\left\langle 3^{7}, 288\right\rangle=: \mathfrak{M}$ for type E.6, resp. two descendants $\left\langle 3^{7}, 289 / 290\right\rangle$ for type E.14. The bifurcation at the vertex $\left\langle 3^{6}, 49\right\rangle$ with nuclear rank two leads to a unique non-metabelian descendant with path $R \leftarrow\left\langle 3^{3}, 3\right\rangle \leftarrow\left\langle 3^{5}, 6\right\rangle \leftarrow\left\langle 3^{6}, 49\right\rangle \leftarrow\left\langle 3^{8}, 616\right\rangle=: G$ for type E.6, resp. two descendants $\left\langle 3^{8}, 617 / 618\right\rangle$ for type E.14 The cover of $\mathfrak{M}=G_{3}^{2} K$ is non-trivial but very simple, since it contains two elements $\operatorname{cov}(\mathfrak{M})=\{\mathfrak{M}, G\}$ only. The decision whether $\ell_{3} K=2$ and $G_{3}^{3} K=\mathfrak{M}$ or $\ell_{3} K=3$ and $\mathrm{G}_{3}^{3} K=G$ requires the iterated IPADs of second order $\tau^{(2)}$ of $\mathfrak{M}$ and $G$, which are listed in Table 3 . The general form $\mathrm{A}(3, c-1) \times C_{3}$ of the component of $\tau^{(2)}$ which corresponds to the commutator subgroup $\mathfrak{M}^{\prime} \simeq G^{\prime} / G^{\prime \prime}$ is a consequence of [[24], Thm. 8.8, p.461], since in terms of the nilpotency class $c$ and coclass $r=2$ of $\mathfrak{M}$ we have $m-2=c-1$ and $e-2=r-1$.

The proof of Theorem 7.7, immediately justifies the following conclusions for $c \leq 5$.

Corollary 7.2. Under the assumptions of Theorem 7.7, the second and third 3-class groups of $K$ are given by their SmallGroups identifier [25] [26], if $c \leq 5$. Independently of $\ell_{3} K$,

if $c=4$, then $\mathrm{G}_{3}^{2} K \simeq\left\langle 3^{6}, 51\right\rangle$ for type E.6, resp. $\left\langle 3^{6}, 50\right\rangle$ for type E.14, and if $c=5$, then $G_{3}^{2} K \simeq\left\langle 3^{7}, 288\right\rangle$ for type E.6, resp. $\left\langle 3^{7}, 298 / 290\right\rangle$ for type E.14.

In the case of a 3 -class tower $\mathrm{F}_{3}^{\infty} \mathrm{K}$ of length $\ell_{3} K=3$, 
if $c=4$, then $G_{3}^{2} K \simeq\left\langle 3^{7}, 293\right\rangle$ for type E.6, resp. $\left\langle 3^{7}, 292\right\rangle$ for type E.14, and

if $c=5$, then $\mathrm{G}_{3}^{2} K \simeq\left\langle 3^{8}, 616\right\rangle$ for type E.6, resp. $\left\langle 3^{8}, 617 / 618\right\rangle$ for type E.14.

The range $0<d<10^{7}$ of fundamental discriminants $d$ of real quadratic fields $K=\mathbb{Q}(\sqrt{d})$ of type $\mathrm{E}$, which underlies Theorem 7.8 in this section, resp. 7.12 in the next section, is just sufficient to prove that each of the possible groups $G$ in Theorem 7.5, resp. 7.9, is actually realized by the 3-tower group $G_{3}^{\infty} K$ of some field $K$.

Proposition 7.2. (Fields $\mathbb{Q}(\sqrt{d})$ with IPOD of type E.6 or E.14 for $0<d<10^{7}$ [5] [24].) In the range $0<d<10^{7}$ of fundamental discriminants $d$ of real quadratic fields $K=\mathbb{Q}(\sqrt{d})$, there exist precisely 3, resp. 4, cases with 3-principalization type E.6, $\varkappa_{1} K \sim(1313)$, resp. E.14, $\varkappa_{1} K \sim(2313)$.

Proof. The results of [[24], Tbl. 6.5, p. 452], where the entry in the last column freq. should be 28 instead of 29 in the first row and 4 instead of 3 in the fourth row, were computed in 2010 by means of the free number theoretic computer algebra system PARI/GP [14] using an implementation of our own principalization algorithm in a PARI script, as described in detail in [[24], \$5, pp. 446-450]. The accumulated frequency 7 for the second and third row was recently split into 3 and 4 with the aid of the computational algebra system MAGMA [17]. See also [[5], Tbl. 4, p. 498].

Remark 7.3. The minimal discriminant $d=5264069$ of real quadratic fields $K=\mathbb{Q}(\sqrt{d})$ of type E.6, resp. $d=3918837$ of type E.14, is indicated in boldface font adjacent to an oval surrounding the vertex, resp. batch of two vertices, which represents the associated second 3-class group $G_{3}^{2} K$, on the branch $\mathcal{B}(6)$ of the coclass tree $\mathcal{T}\langle 243,6\rangle$ in Figure 3.

Theorem 7.8. (3-Class towers $\mathrm{F}_{3}^{\infty} \mathbb{Q}(\sqrt{d})$ with IPOD of type E.6 or E. 14 for $\left.0<d<10^{7}\right)$ Among the 3 real quadratic fields $K=\mathbb{Q}(\sqrt{d})$ with IPOD of type E.6 in Proposition 7.2,

- the 2 fields $(67 \%)$ with discriminants

$$
d \in\{5264069,6946573\}
$$

have the unique 3-class tower group $G \simeq\left\langle 3^{8}, 616\right\rangle$ and 3-tower length $\ell_{3} K=3$,

- the single field ( $33 \%$ ) with discriminant

$$
d=7153097
$$

has the unique 3-class tower group $G \simeq\left\langle 3^{7}, \mathbf{2 8 8}\right\rangle$ and 3-tower length $\ell_{3} K=\mathbf{2}$. Among the 4 real quadratic fields $K=\mathbb{Q}(\sqrt{d})$ with IPOD of type E.14 in Proposition 7.2,

- the 3 fields ( $75 \%$ ) with discriminants

$$
d \in\{3918837,8897192,9991432\}
$$

have 3-class tower group $G \simeq\left\langle 3^{7}, \mathbf{2 8 9}\right\rangle$ or $G \simeq\left\langle 3^{7}, \mathbf{2 9 0}\right\rangle$ and 3-tower length $\ell_{3} K=2$,

- the single field ( $25 \%$ ) with discriminant 
$d=9433849$

has 3-class tower group $G \simeq\left\langle 3^{8}, 617\right\rangle$ or $G \simeq\left\langle 3^{8}, 618\right\rangle$ and 3-tower length $\ell_{3} K=3$.

Proof. Since all these real quadratic fields $K=\mathbb{Q}(\sqrt{d})$ have 3-capitulation type $\varkappa_{1} K=(1122)$ or $(3122)$ and $1^{\text {st }}$ IPAD $\tau^{(1)} K=\left[1^{2} ; 32,1^{3},(21)^{2}\right]$, and the 4 fields with $d \in\{3918837,7153097,8897192\}$ have $2^{\text {nd }}$ IPAD

$\tau_{1} L_{1}=\left(2^{2} 1,\left(31^{2}\right)^{3}\right), \tau_{1} L_{2}=\left(2^{2} 1,\left(\mathbf{1}^{3}\right)^{3},\left(1^{2}\right)^{9}\right), \tau_{1} L_{3}=\left(2^{2} 1,(\mathbf{2 1})^{3}\right), \tau_{1} L_{4}=\left(2^{2} 1,(\mathbf{2 1})^{3}\right)$,

whereas the 3 fields with $d \in\{5264069,6946573,9433849\}$ have $2^{\text {nd }}$ IPAD

$\tau_{1} L_{1}=\left(2^{2} 1,\left(31^{2}\right)^{3}\right), \tau_{1} L_{2}=\left(2^{2} 1,\left(\mathbf{2 1}^{2}\right)^{3},\left(1^{2}\right)^{9}\right), \tau_{1} L_{3}=\left(2^{2} 1,(\mathbf{3 1})^{3}\right), \tau_{1} L_{4}=\left(2^{2} 1,(31)^{3}\right)$,

the claim is a consequence of Theorem 7.5.

Remark 7.4. The computation of the 3-principalization type E.14 of the field with $d=9433849$ resisted all attempts with MAGMA versions up to V2.21-7. Due to essential improvements in the change from relative to absolute number fields, made by the staff of the Computational Algebra Group at the University of Sydney, it actually became feasible to figure it out with V2.21-8 [17] for UNIX/LINUX machines or V2.22-3 for any operating system.

\subsection{3-Groups $G$ of Coclass cc $(G)=2$ Arising from $\left\langle 3^{5}, 8\right\rangle$}

Table 4 shows the designation of the transfer kernel type, the IPOD $\varkappa_{1} G$, and the iterated multi-layered IPAD of second order,

$$
\tau_{*}^{(2)} G=\left[\tau_{0} G ;\left[\tau_{0} H ; \tau_{1} H ; \tau_{2} H\right]_{H \in \mathrm{Lyr}_{1} G}\right],
$$

for 3-groups $G$ on the coclass tree $\mathcal{T}^{2}\left\langle 3^{5}, 8\right\rangle$ up to order $|G|=3^{8}$, characterized by the logarithmic order, lo, and the SmallGroup identifier, id [25] [26]. To enable a brief reference for relative identifiers we put $U:=\left\langle 3^{6}, 54\right\rangle$, since this group was called the non-CF group $U$ by Ascione [38] [39].

The groups in Table 4 are represented by vertices of the tree diagram in Figure 4.

Theorem 7.9. (Smallest possible 3-tower groups $G=\mathrm{G}_{3}^{\infty} \mathrm{K}$ of type E.8 or E.9 [1]) Let $G$ be a finite 3-group with IPAD of first order $\tau^{(1)} G=\left[\tau_{0} G ; \tau_{1} G\right]$, where $\tau_{0} G=1^{2}$ and $\tau_{1} G=\left(21,32,(21)^{2}\right)$ is given in ordered form.

If the IPOD of $G$ is of type E.8, $\varkappa_{1} G=(2234)$, resp. E.9, $\varkappa_{1} G=(2334) \sim(2434)$, then the IPAD of second order $\tau^{(2)} G=\left[\tau_{0} G ;\left(\tau_{0} H_{i} ; \tau_{1} H_{i}\right)_{1 \leq i \leq 4}\right]$, where the maximal subgroups of index 3 in $G$ are denoted by $H_{1}, \cdots, H_{4}$, determines the isomorphism type of $G$ in the following way:

1) $\tau^{(1)} H_{i}=\left[21 ; 2^{2} 1,(21)^{3}\right]$ for $i \in\{1,3,4\}$

if and only if $G \simeq\left\langle 3^{7}, 304\right\rangle$, resp. $G \simeq\left\langle 3^{7}, 302\right\rangle$ or $G \simeq\left\langle 3^{7}, 306\right\rangle$,

2) $\tau^{(1)} H_{i}=\left[21 ; 2^{2} 1,(31)^{3}\right]$ for $i \in\{1,3,4\}$

if and only if $G \simeq\left\langle 3^{8}, 622\right\rangle$, resp. $G \simeq\left\langle 3^{8}, 620\right\rangle$ or $G \simeq\left\langle 3^{8}, 624\right\rangle$, whereas the component $\tau^{(1)} H_{2}=\left[32 ; 2^{2} 1,\left(31^{2}\right)^{3}\right]$ is fixed and does not admit a 
distinction.

Proof. This is essentially [[1], Thm. 6.3, pp. 298-299]. It is also an immediate consequence of Table 4, which has been computed with MAGMA [17]. As a termination criterion we can now use the more precise [[4], Thm. 5.1] instead of [[7], Cor. 3.0.1, p. 771].

Figure 4 visualizes 3-groups which arise as second 3-class groups $\mathfrak{M}=\mathrm{G}_{3}^{2} K$ of real quadratic fields $K=\mathbb{Q}(\sqrt{d}), d>0$, with 3-principalization types E.8 and E.9 in section $\$ 7.6$ and the corresponding minimal discriminants.

\subsection{Parametrized IPADs of Second Order for the Coclass Tree $\mathcal{T}^{2}\left\langle 3^{5}, 8\right\rangle$}

Let $G \in \mathcal{T}^{2}\left\langle 3^{5}, 8\right\rangle$ be a descendant of coclass $\operatorname{cc}(G)=2$ of the root $\left\langle 3^{5}, 8\right\rangle$. Denote by $c:=\operatorname{cl}(G)$ the nilpotency class of $G$, by $t:=\operatorname{dl}((G)-2$ the indicator of a three-stage group, and by $k:=k(G)$, resp $k:=k(\pi G)$, the defect of commutativity of $G$ itself if $t=0$, and of the metabelian parent $\pi G$ if $t=1$.

Theorem 7.10. In dependence on the parameters $c$, $t$ and $k$, the IPAD of second order of $G$ has the form

$$
\begin{aligned}
\tau^{(2)} G= & 1^{2} ;\left(\mathrm{A}(3, c-k-t) ; \mathrm{A}(3, c-1-k-t) \times C_{3},\left(\mathrm{~B}(3, c-1-k-t) \times C_{3}\right)^{3}\right), \\
& \left(21 ; \mathrm{A}(3, c-1-k-t) \times C_{3},(21)^{3}\right)^{3},
\end{aligned}
$$

where a variant $\mathrm{B}(3, n)$ of the nearly homocyclic abelian 3-group $\mathrm{A}(3, n)$ of order $n \geq 2$ is defined as in Formula (7.4) of Theorem 7.6.

\subsection{Number Fields with IPOD of Type E.8 or E.9}

Let $K$ be a number field with 3-class group $\mathrm{Cl}_{3} K \simeq C_{3} \times C_{3}$ and first layer $\operatorname{Lyr}_{1} K=\left\{L_{1}, \cdots, L_{4}\right\}$ of unramified abelian extensions.

Theorem 7.11. (Criteria for $\ell_{3} K \in\{2,3\}$.) Let the IPOD of $K$ be of type E.8, $\varkappa_{1} K \sim(1231)$, resp. E.9, $\varkappa_{1} K \sim(2231)$. If $\tau_{1} K \sim(\mathrm{A}(3, c), 21,21,21)$ with $c \geq 4$, then

- $\ell_{3} K=2 \Leftrightarrow \tau_{1} L_{j} \sim\left(\mathrm{A}(3, c-1) \times C_{3},(\mathbf{2 1})^{3}\right)$ for $2 \leq j \leq 4$,

- $\ell_{3} K=3 \Leftrightarrow \tau_{1} L_{j} \sim\left(\mathrm{A}(3, c-1) \times C_{3},(31)^{3}\right)$ for $2 \leq j \leq 4$.

Proof. Exemplarily, we conduct the proof for $c=5$, which is the most important situation for our computational applications.

Searching for the Artin pattern $\operatorname{AP}_{1}=\left(\tau_{1}, \varkappa_{1}\right)$ with $\tau_{1} \sim(32,21,21,21)$ and $\varkappa_{1} \sim(1231)$, resp. $(2231)$, in the descendant tree $\mathcal{T}(R)$ with abelian root $R:=\left\langle 3^{2}, 2\right\rangle \simeq C_{3} \times C_{3}$, unambiguously leads to the unique metabelian descendant with path $R \leftarrow\left\langle 3^{3}, 3\right\rangle \leftarrow\left\langle 3^{5}, 8\right\rangle \leftarrow\left\langle 3^{6}, 54\right\rangle \leftarrow\left\langle 3^{7}, 304\right\rangle=: \mathfrak{M}$ for type E.8, resp. two descendants $\left\langle 3^{7}, 302 / 306\right\rangle$ for type E.9. The bifurcation at the vertex $\left\langle 3^{6}, 54\right\rangle$ with nuclear rank two leads to a unique non-metabelian descendant with path $R \leftarrow\left\langle 3^{3}, 3\right\rangle \leftarrow\left\langle 3^{5}, 8\right\rangle \leftarrow\left\langle 3^{6}, 54\right\rangle \leftarrow\left\langle 3^{8}, 622\right\rangle=$ : $G$ for type E.8, resp. two descendants $\left\langle 3^{8}, 620 / 624\right\rangle$ for type E.9. The cover of $\mathfrak{M}=G_{3}^{2} K$ is 
non-trivial but very simple, since it contains two elements $\operatorname{cov}(\mathfrak{M})=\{\mathfrak{M}, G\}$ only. The decision whether $\ell_{3} K=2$ and $G_{3}^{3} K=\mathfrak{M}$ or $\ell_{3} K=3$ and $\mathrm{G}_{3}^{3} K=G$ requires the iterated IPADs of second order $\tau^{(2)}$ of $\mathfrak{M}$ and $G$, which are listed in Table 4. The general form $\mathrm{A}(3, c-1) \times C_{3}$ of the component of $\tau^{(2)}$ which corresponds to the commutator subgroup $\mathfrak{M}^{\prime} \simeq G^{\prime} / G^{\prime \prime}$ is a consequence of [[24], Thm. 8.8, p. 461], since in terms of the nilpotency class $c$ and coclass $r=2$ of $\mathfrak{M}$ we have $m-2=c-1$ and $e-2=r-1$.

The proof of Theorem 7.11, immediately justifies the following conclusions for $c \leq 5$.

Corollary 7.3. Under the assumptions of Theorem 7.11, the second and third 3-class groups of $K$ are given by their SmallGroups identifier [25] [26], if $c \leq 5$. Independently of $\ell_{3} K$,

if $c=4$, then $\mathrm{G}_{3}^{2} K \simeq\left\langle 3^{6}, 55\right\rangle$ for type E.8, resp. $\left\langle 3^{6}, 53\right\rangle$ for type E.9, and

if $c=5$, then $G_{3}^{2} K \simeq\left\langle 3^{7}, 304\right\rangle$ for type E.8, resp. $\left\langle 3^{7}, 302 / 306\right\rangle$ for type E.9.

In the case of a 3 -class tower $\mathrm{F}_{3}^{\infty} \mathrm{K}$ of length $\ell_{3} \mathrm{~K}=3$,

if $c=4$, then $\mathrm{G}_{3}^{3} K \simeq\left\langle 3^{7}, 309\right\rangle$ for type E.8, resp. $\left\langle 3^{7}, 300\right\rangle$ for type E.9, and

if $c=5$, then $G_{3}^{3} K \simeq\left\langle 3^{8}, 622\right\rangle$ for type E.8, resp. $\left\langle 3^{8}, 620 / 624\right\rangle$ for type E.9.

Proposition 7.3. (Fields $\mathbb{Q}(\sqrt{d})$ with IPOD of type E.8 or E.9 for $0<d<10^{7}$ [5] [24]) In the range $0<d<10^{7}$ of fundamental discriminants $d$ of real quadratic fields $K=\mathbb{Q}(\sqrt{d})$, there exist precisely 3, resp. 11, cases with 3-principalization type E.8, $\varkappa_{1} K \sim(1231)$, resp. E.9, $\varkappa_{1} K \sim(2231)$.

Proof. The results of [[24], Tbl. 6.7, p. 453] were computed in 2010 by means of PARI/GP [14] using an implementation of our principalization algorithm, as described in [[24], 5, pp. 446-450]. The accumulated frequency 14 in the last column freq.for the second and third row was recently split into 3 and 11 with the aid of MAGMA [17]. See also [[5], Tbl. 4, p. 498].

Remark 7.5. The minimal discriminant $d=6098360$ of real quadratic fields $K=\mathbb{Q}(\sqrt{d})$ of type E.8, resp. $d=342664$ of type E.9, is indicated in boldface font adjacent to an oval surrounding the vertex, resp. batch of two vertices, which represents the associated second 3-class group $G_{3}^{2} K$, on the branch $\mathcal{B}(6)$ of the coclass tree $\mathcal{T}^{2}\langle 243,8\rangle$ in Figure 4.

Theorem 7.12. (3-Class towers $\mathrm{F}_{3}^{\infty} \mathbb{Q}(\sqrt{d})$ with IPOD of type E.8 or E.9 for $0<d<10^{7}$ ) Among the 3 real quadratic fields $K=\mathbb{Q}(\sqrt{d})$ with IPOD of type E.8 in Proposition 7.3,

- the 2 fields ( $67 \%$ ) with discriminants

$$
d \in\{6098360,7100889\}
$$

have the unique 3-class tower group $G \simeq\left\langle 3^{8}, 622\right\rangle$ and 3-tower length $\ell_{3} K=3$,

- the single field (33\%) with discriminant

$$
d=8632716
$$


has the unique 3-class tower group $G \simeq\left\langle 3^{7}, \mathbf{3 0 4}\right\rangle$ and 3-tower length $\ell_{3} K=\mathbf{2}$. Among the 11 real quadratic fields $K=\mathbb{Q}(\sqrt{d})$ with IPOD of type E.9 in Proposition 7.3,

- the 7 fields ( $64 \%$ ) with discriminants

$d \in\{342664,1452185,1787945,4861720,5976988,8079101,9674841\}$

have 3-class tower group $G \simeq\left\langle 3^{8}, \mathbf{6 2 0}\right\rangle$ or $G \simeq\left\langle 3^{8}, \mathbf{6 2 4}\right\rangle$ and 3 -tower length $\ell_{3} K=\mathbf{3}$,

- the 4 fields ( $36 \%$ ) with discriminants

$$
d \in\{4760877,6652929,7358937,9129480\}
$$

have 3-class tower group $G \simeq\left\langle 3^{7}, 302\right\rangle$ or $G \simeq\left\langle 3^{7}, 306\right\rangle$ and 3 -tower length $\ell_{3} K=2$.

Proof. Since all these real quadratic fields $K=\mathbb{Q}(\sqrt{d})$ have 3-capitulation type $\varkappa_{1} K=(2234)$ or $(2334)$ and $1^{\text {st }} \operatorname{IPAD} \tau^{(1)} K=\left[1^{2} ; 32,(21)^{3}\right]$, and the 5 fields with $d \in\{4760877,6652929,7358937,8632716,9129480\}$ have $2^{\text {nd }} I P A D$

$$
\tau_{1} L_{1}=\left(2^{2} 1,\left(31^{2}\right)^{3}\right), \tau_{1} L_{2}=\left(2^{2} 1,(\mathbf{2 1})^{3}\right), \tau_{1} L_{3}=\left(2^{2} 1,(\mathbf{2 1})^{3}\right), \tau_{1} L_{4}=\left(2^{2} 1,(\mathbf{2 1})^{3}\right),
$$

whereas the 9 fields with $d \in\{342664,1452185,1787945,4861720,5976988$, 6098360, 7100889, 8079101, 9674841\}

have $2^{\text {nd }}$ IPAD

$$
\tau_{1} L_{1}=\left(2^{2} 1,\left(31^{2}\right)^{3}\right), \tau_{1} L_{2}=\left(2^{2} 1,(31)^{3}\right), \tau_{1} L_{3}=\left(2^{2} 1,(31)^{3}\right), \tau_{1} L_{4}=\left(2^{2} 1,(31)^{3}\right),
$$

the claim is a consequence of Theorem 7.9.

Remark 7.6. The 3-principalization type E.9 of the field with $d=9674841$ could not be computed with MAGMA versions up to V2.21-7. Finally, we succeeded to figure it out by means of V2.21-8 [17].

Figure 5 visualizes sporadic 3 -groups of section $\$ 7.9$ which arise as second 3-class groups $\mathfrak{M}=\mathrm{G}_{3}^{2} K$ of real quadratic fields $K=\mathbb{Q}(\sqrt{d}), d>0$, with 3-principalization types D.10, D.5, G.19 and H.4 and the corresponding minimal discriminants, resp. absolute frequencies, which are given in section $\$ 7.10$ and $\$ 7.12$.

\subsection{Sporadic 3-Groups $G$ of Coclass $\operatorname{cc}(G)=2$}

Table 5 shows the designation of the transfer kernel type, the IPOD $\varkappa_{1} G$, and the iterated multi-layered IPAD of second order,

$$
\tau_{*}^{(2)} G=\left[\tau_{0} G ;\left[\tau_{0} H ; \tau_{1} H ; \tau_{2} H\right]_{H \in \mathrm{Ly}_{1} G}\right],
$$

for sporadic 3-groups $G$ of type H.4 up to order $|G|=3^{8}$, characterized by the logarithmic order, lo, and the SmallGroup identifier, id [25] [26]. To enable a brief reference for relative identifiers we put $N:=\left\langle 3^{6}, 45\right\rangle$, since this group was called the non-CF group $N$ by Ascione [38] [39].

The groups in Table 5 are represented by vertices of the tree diagram in Figure 6. Figure 6 visualizes sporadic 3 -groups of section $\$ 7.9$ which arise as 
3-class tower groups $G=G_{3}^{\infty} K$ of real quadratic fields $K=\mathbb{Q}(\sqrt{d}), d>0$, with 3-principalization type H.4 and the corresponding minimal discriminants, resp. absolute frequencies, in Theorem 7.13 and 7.14.

The tree is infinite, according to Bartholdi, Bush [45] and [[1], Cor. 6.2, p. 301].

For $d=+2852733$ and $d=-6583$, we can only give the conjectural location of $G$.

\subsection{Real Quadratic Fields of Type H.4}

Proposition 7.4 (Fields of type H.4 up to $d<10^{7} \quad$ [5] [24])

In the range $0<d<10^{7}$ of fundamental discriminants $d$ of real quadratic fields $K=\mathbb{Q}(\sqrt{d})$, there exist precisely 27 cases with 3-principalization type H.4, $\varkappa_{1} K=(4111)$, and IPAD $\tau^{(1)} K=\left[1^{2} ;\left(1^{3}\right)^{3}, 21\right]$. They share the common second 3 -class group $\mathrm{G}_{3}^{2} K \simeq\left\langle 3^{6}, 45\right\rangle$.

Proof. The results of [[24], Tbl. 6.3, p. 452] were computed in 2010 by means of PARI/GP [14] using an implementation of our principalization algorithm, as described in [[24], 5, pp. 446-450]. The frequency 27 in the last column "freq." for the fourth row concerns type H.4.

Remark 7.7. To discourage any misinterpretation, we point out that there are four other real quadratic fields $K=\mathbb{Q}(\sqrt{d})$ with discriminants $d \in\{1162949,2747001,3122232,4074493\}$ in the range $0<d<10^{7}$ which possess the same 3-principalization type H.4. However their second 3-class group $G_{3}^{2} K$ is isomorphic to either $\left\langle 3^{7}, 286\right\rangle-\# 1 ; 2$ or $\left\langle 3^{7}, 287\right\rangle-\# 1 ; 2$ of order $3^{8}$, which is not a sporadic group but is located on the coclass tree $\mathcal{T}^{2}\left\langle 3^{5}, 6\right\rangle$, and has a different IPAD $\tau^{(1)} K=\left[1^{2} ; 32,1^{3},(21)^{2}\right]$. The 3-class towers of these fields are determined in [20].

Theorem 7.13. (3-Class towers of type H.4 up to $d<10^{7}$ )

Among the 27 real quadratic fields $K=\mathbb{Q}(\sqrt{d})$ with type H.4 in Proposition 7.4 ,

- the 11 fields (41\%) with discriminants

$$
\begin{aligned}
d \in & \{957013,1571953,1734184,3517689,4025909,4785845, \\
& 4945973,5562969,5562969,6318733,7762296,8070637\}
\end{aligned}
$$

have the unique 3-class tower group $G \simeq\left\langle 3^{7}, 273\right\rangle$ and 3-tower length $\ell_{3} K=3$,

- the 8 fields ( $29 \%$ ) with discriminants

$$
\begin{array}{r}
d \in\{2023845,4425229,6418369,6469817, \\
\text { 6775224, 6895612, 7123493, 9419261 }\}
\end{array}
$$

have 3-class tower group $G \simeq\left\langle 3^{7}, 271\right\rangle$ or $G \simeq\left\langle 3^{7}, 272\right\rangle$ and 3 -tower length $\ell_{3} K=\mathbf{3}$,

- the 5 fields ( $19 \%$ ) with discriminants

$$
d \in\{2303112,3409817,3856685,5090485,6526680\}
$$


have the unique 3-class tower group $G \simeq\left\langle 3^{7}, 270\right\rangle$ and 3-tower length $\ell_{3} K=3$,

- the 3 fields ( $11 \%)$ with discriminants

$$
d \in\{2852733,8040029,8369468\}
$$

have a 3 -class tower group of order at least $3^{8}$ and 3 -tower length $\ell_{3} K \in\{3,4, \cdots\}$.

Note that $\left\langle 3^{7}, \mathbf{2 7 0}\right\rangle=\left\langle 3^{6}, 45\right\rangle-\# 1 ; \mathbf{1},\left\langle 3^{7}, \mathbf{2 7 1}\right\rangle=\left\langle 3^{6}, 45\right\rangle-\# 1 ; \mathbf{2}$, $\left\langle 3^{7}, \mathbf{2 7 2}\right\rangle=\left\langle 3^{6}, 45\right\rangle-\# 1 ; 3$, and $\left\langle 3^{7}, 273\right\rangle=\left\langle 3^{6}, 45\right\rangle-\# 1 ; 4$.

Proof. Extensions of absolute degrees 6 and 18 were constructed in steps with MAGMA [17], using the class field package of C. Fieker [46]. The resulting iterated IPADs of second order $\tau^{(2)} K$ were used for the identification, according to Table 5, which is also contained in the more extensive theorem [[1], Thm. 6.5, pp. 304-306].

\subsection{Imaginary Quadratic Fields of Type H.4}

Proposition 7.5. (Fields of type H.4 down to $d>-3 \times 10^{4}$ [5] [47])

In the range $-30000<d<0$ of fundamental discriminants $d$ of imaginary quadratic fields $K=\mathbb{Q}(\sqrt{d})$, there exist precisely 6 cases with 3-principalization type H.4, $\varkappa_{1} K=(4111)$, and IPAD $\tau^{(1)} K=\left[1^{2} ;\left(1^{3}\right)^{3}, 21\right]$. They share the common second 3 -class group $G_{3}^{2} K \simeq\left\langle 3^{6}, 45\right\rangle$.

Proof. In the table of suitable base fields [[47], p. 84], the row Nr. 4 contains 7 discriminants $-30000<d<0$ of imaginary quadratic fields $K=\mathbb{Q}(\sqrt{d})$ with type H.4. It was computed in 1989 by means of our implementation of the principalization algorithm by Scholz and Taussky, described in [[47], pp. 80-83]. In 1989 already, we recognized that only for the discriminant $d=-21668$ one of the four absolute cubic subfields $L_{i}, 1 \leq i \leq 4$, of the unramified cyclic cubic extensions $N_{i}$ of $K$ has 3 -class number $h_{3} L_{i}=9$, which is not the case for the other 6 cases of type H.4 in the table [[47], pp. 78-79]. According to [[5], Prop. 4.4, p. 485] or [[5], Thm. 4.2, p. 489] or [48], the exceptional cubic field $L_{i}$ is contained in a sextic field $N_{i}$ with 3-class number $h_{3} N_{i}=3 \times\left(h_{3} L_{i}\right)^{2}=243$, which discourages an IPAD $\tau^{(1)} K=\left[1^{2} ;\left(1^{3}\right)^{3}, 21\right]$.

Remark 7.8. The imaginary quadratic field with discriminant $d=-21668$ possesses the same 3-principalization type H.4, but its second 3-class group $\mathrm{G}_{3}^{2} K$ is isomorphic to either $\left\langle 3^{7}, 286\right\rangle-\# 1 ; 2$ or $\left\langle 3^{7}, 287\right\rangle-\# 1 ; 2$ of order $3^{8}$, and has the different IPAD $\tau^{(1)} K=\left[1^{2} ; 32,1^{3},(21)^{2}\right]$. Results for this field will be given in [20].

Theorem 7.14. (3-Class towers of type H.4 down to $d>-3 \times 10^{4}$ )

Among the 6 imaginary quadratic fields $K=\mathbb{Q}(\sqrt{d})$ with type H.4 in Proposition 7.5,

- the 3 fields ( $\mathbf{5 0} \%$ ) with discriminants

$$
d \in\{-3896,-25447,-27355\}
$$

have the unique 3-class tower group $G \simeq\left\langle 3^{8}, 606\right\rangle$ and 3 -tower length $\ell_{3} K=3$,

- the 3 fields ( $\mathbf{5 0} \%$ ) with discriminants 


$$
d \in\{-6583,-23428,-27991\}
$$

have a 3-class tower group of order at least $3^{11}$ and 3-tower length $\ell_{3} K \in\{3,4, \cdots\}$.

Proof. Using the technique of Fieker [46], extensions of absolute degrees 6 and 54 were constructed in two steps, squeezing MAGMA [17] close to its limits. The resulting multi-layered iterated IPADs of second order $\tau_{*}^{(2)} K$ were used for the identification, according to Table 5, resp. the more detailed theorem [[1], Thm. 6.5, pp. 304-306].

Table 6 shows the designation of the transfer kernel type, the IPOD $\varkappa_{1} G$, and the iterated multi-layered IPAD of second order,

$$
\tau_{*}^{(2)} G=\left[\tau_{0} G ;\left[\tau_{0} H ; \tau_{1} H ; \tau_{2} H\right]_{H \in \mathrm{Lyr}_{1} G}\right],
$$

for sporadic 3-groups $G$ of type G.19 up to order $|G|=3^{14}$, characterized by the logarithmic order, lo, and the SmallGroup identifier, id [25] [26], resp. the relative identifier for $l o \geq 9$. To enable a brief reference for relative identifiers we put

$W:=\left\langle 3^{6}, 57\right\rangle$, since this group was called the non-CF group $W$ by Ascione [39] [38],

$$
\begin{aligned}
& \Phi:=\left\langle 3^{8}, 626\right\rangle, \quad \Psi:=\left\langle 3^{8}, 628\right\rangle, \text { and further } \\
& Y:=\left\langle 3^{8}, 629\right\rangle, \quad Y_{1}:=Y-\# 1 ; 2, \text { and } \\
& Z:=\left\langle 3^{8}, 630\right\rangle, \quad Z_{1}:=Z-\# 1 ; 2, \quad Z_{2}:=Z-\# 2 ; 7 .
\end{aligned}
$$

The groups in Table 6 are represented by vertices of the tree diagram in Figure 7. Figure 7 visualizes sporadic 3-groups of Table 6 which arise as 3-class tower groups $G=\mathrm{G}_{3}^{\infty} K$ of real quadratic fields $K=\mathbb{Q}(\sqrt{d}), d>0$, with 3principalization type $\mathrm{G} .19$ and the corresponding minimal discriminants, resp. absolute frequencies in Theorem 7.16 and 7.17.

The subtrees $\mathcal{T}(W-\# 2 ; i)$ are finite and drawn completely for $i \in\{1,3,5\}$, but they are omitted in the complicated cases $i \in\{2,4,6\}$, where they reach beyond order $3^{20}$.

For $d=+24126593,-12067$ and -54195 , we can only give the conjectural location of $G$.

\subsection{Real Quadratic Fields of Type G.19}

Proposition 7.6 (Fields of type G.19 up to $d<10^{7}$ [5] [24])

In the range $0<d<10^{7}$ of fundamental discriminants $d$ of real quadratic fields $K=\mathbb{Q}(\sqrt{d})$, there exist precisely 11 cases with 3-principalization type G.19, $\varkappa_{1} K=(2143)$, consisting of two disjoint 2-cycles. Their IPAD is uniformly given by $\tau^{(1)} K=\left[1^{2} ;(21)^{4}\right]$, in this range.

Proof. The results of [[24], Tbl. 6.3, p. 452] were computed in 2010 by means of PARI/GP [14] using an implementation of our principalization algorithm, as described in [[24], 5, pp. 446-450]. The frequency 11 in the last column freq.of the first row concerns type G.19.

Theorem 7.15. (3-Class towers of type G.19 up to $d<10^{7}$ )

The 11 real quadratic fields $K=\mathbb{Q}(\sqrt{d})$ in Proposition 7.6 with dis- 
criminants

$$
\begin{aligned}
d \in\{214712,943077,1618493,2374077,3472653,4026680, \\
\\
4628117,5858753,6405317,7176477,7582988\}
\end{aligned}
$$

have the unique 3-class tower group $G \simeq\left\langle 3^{7}, 311\right\rangle=\left\langle 3^{6}, 57\right\rangle-\# 1 ; 1$ and 3-tower length $\ell_{3} K=\mathbf{3}$.

Proof. Extensions of absolute degrees 6 and 18 were constructed with MAGMA [17], using Fieker's class field package [46]. The resulting uniform iterated IPAD of second order $\tau^{(2)} K=\left[1^{2} ;\left(21 ; 1^{4},\left(21^{2}\right)^{3}\right),\left(21 ; 1^{4},(\mathbf{2 1})^{3}\right)^{3}\right]$ was used for the identification of $G$, according to Table 6 .

Since real quadratic fields of type G.19 seemed to have a very rigid behaviour with respect to their 3-class field tower, admitting no variation at all, we were curious about the continuation of these discriminants beyond the range $d<10^{7}$. Fortunately, M. R. Bush granted access to his extended numerical results for $d<10^{9} \quad$ [42], and so we are able to state the following unexpected answer to our question "Is the 3-class tower group $G$ of real quadratic fields with type G.19 and IPAD $\tau^{(1)}(K)=\left[1^{2} ;(21)^{4}\right]$ always isomorphic to $\left\langle 3^{7}, \mathbf{3 1 1}\right\rangle$ in the SmallGroups Library?"

Proposition 7.7. (Fields of type G.19 up to $d<5 \times 10^{7}$ [42]) In the range $0<d<5 \times 10^{7}$ of fundamental discriminants $d$ of real quadratic fields $K=\mathbb{Q}(\sqrt{d})$, there exist precisely 64 cases with 3-principalization type G.19, $\varkappa_{1} K=(2143)$, and with IPAD $\tau^{(1)} K=\left[1^{2} ;(21)^{4}\right]$.

Proof. Private communication by M. R. Bush [42].

Theorem 7.16. (3-Class towers of type G.19 up to $d<5 \times 10^{7}$ )

Among the 64 real quadratic fields $K=\mathbb{Q}(\sqrt{d})$ with type $\mathrm{G} .19$ in Proposition 7.7,

- the 11 fields with discriminants $d$ in Theorem 7.15 and the 44 fields with discriminants

$$
\begin{aligned}
& d \in\{10169729,11986573,14698056,14836573,16270305,16288424 \text {, } \\
& \text { 18195889, 19159368, 21519660, 21555097, 22296941, 22431068, } \\
& \text { 24229337, 25139461, 26977089, 27696973, 29171832, 29523765, } \\
& \text { 30019333, 31921420, 32057249, 33551305, 35154857, 35846545, } \\
& \text { 36125177, 36409821, 37344053, 37526493, 37796984, 38691433, } \\
& \text { 39693865, 40875944, 42182968, 42452445, 42563029, 43165432, } \\
& \text { 43934584, 44839889, 44965813, 45049001, 46180124, 46804541, } \\
& \text { 46971381, 48628533\} }
\end{aligned}
$$

(that is, together 55 fields or $86 \%$ ) have $\tau^{(2)} K=\left[1^{2} ;\left(21 ; 1^{4},\left(21^{2}\right)^{3}\right),\left(21 ; 1^{4},(21)^{3}\right)^{3}\right]$, the unique 3-class tower group $G \simeq\left\langle 3^{7}, \mathbf{3 1 1}\right\rangle$, and3-tower length $\ell_{3} K=\mathbf{3}$,

- the 3 fields ( $5 \%$ ) with discriminants

$$
d \in\{21974161,22759557,35327365\}
$$

have IPAD of second order $\tau^{(2)} K=\left[1^{2} ;\left(21 ; 1^{4},\left(31^{2}\right)^{3}\right),\left(21 ; 1^{4},\left(2 \mathbf{1}^{2}\right)^{3}\right)^{3}\right]$, the 
unique 3-tower group $G \simeq\left\langle 3^{8}, \mathbf{6 2 9}\right\rangle-\# 1 ; 2-\# 1 ; 1$ of order $3^{10}$, and 3 -tower length $\ell_{3} K=\mathbf{3}$,

- the 6 fields ( $9 \%$ ) with discriminants

$$
d \in\{24126593,29739477,31353229,35071865,40234205,40706677\}
$$

have iterated IPAD of second order $\tau^{(2)} K=\left[1^{2} ;\left(21 ; 1^{4},\left(\mathbf{2 1}^{2}\right)^{3}\right)^{4}\right]$, a 3-class tower group of order at least $3^{8}$, and 3-tower length $\ell_{3} K \in\{3,4, \cdots\}$.

Proof. Similar to the proof of Theorem 7.15, using Table 6, but now applied to the more extensive range of discriminants and various iterated IPADs of second order.

\subsection{Imaginary Quadratic Fields of Type G.19}

Proposition 7.8. (Fields of type G.19 down to $d>-5 \times 10^{5} \quad$ [5] [24])

In the range $-5 \times 10^{5}<d<0$ of fundamental discriminants $d$ of imaginary quadratic fields $K=\mathbb{Q}(\sqrt{d})$, there exist precisely 46 cases with 3-principalization type $\mathrm{G} .19, \varkappa_{1} \mathrm{~K}=(2143)$, consisting of two disjoint 2-cycles, and with IPAD $\tau^{(1)} K=\left[1^{2} ;(21)^{4}\right]$.

Proof. The results of [[24], Tbl. 6.4, p. 452] were computed in 2010 by means of PARI/GP [14] using an implementation of our principalization algorithm, as described in [[24], 5, pp. 446-450]. The frequency 94 in the last column freq.of the first row concerns type G.19 in the bigger range $-10^{6}<d<0$. Reduced to the first half of this range, we have 46 occurrences.

Theorem 7.17. (3-Class towers of type G.19 down to $d>-5 \times 10^{5}$ )

Among the 46 imaginary quadratic fields $K=\mathbb{Q}(\sqrt{d})$ with type $\mathrm{G} .19$ in Proposition 7.8,

- the 30 fields (65\%) with discriminants

$$
\begin{aligned}
& d \in\{-12067, \quad-49924, \quad-60099, \quad-83395, \quad-86551, \quad-93067 \text {, } \\
& -152355,-153771,-161751,-168267,-195080,-235491 \text {, } \\
& -243896,-251723,-283523,-310376,-316259,-337816 \text {, } \\
& -339459,-344823,-350483,-407983,-421483,-431247 \text {, } \\
& -433732,-442367,-444543,-453463,-458724,-471423\}
\end{aligned}
$$

have iterated IPAD of second order $\tau^{(2)} K=\left[1^{2} ;\left(21 ; 1^{4},\left(\mathbf{2 1}^{2}\right)^{3}\right)^{4}\right]$. Conjecturally, most of them have 3 -class tower group $G \simeq\left\langle 3^{8}, 625\right\rangle-\# 1 ; 2-\# 2 ; 1 \mid 2$ of order $3^{11}$, and 3-tower length $\ell_{3} K=\mathbf{3}$, but $|G| \geq 3^{14}$ and $\ell_{3} K \geq 4$ cannot be excluded.

- The 7 fields (15\%) with discriminants

$$
d \in\{-54195,-96551,-104659,-133139,-222392,-313207,-420244\}
$$

have iterated IPAD of second order $\tau^{(2)} K=\left[1^{2} ;\left(21 ; 1^{4},\left(2^{2} \mathbf{1}\right)^{3}\right)^{4}\right]$, a 3-class tower group of order at least $3^{11}$, and 3 -tower length $\ell_{3} K \in\{3,4, \cdots\}$,

- the 7 fields (15\%) with discriminants 
$d \in\{-114936,-118276,-272659,-317327,-328308,-339563,-485411\}$

have iterated IPAD of second order $\tau^{(2)} K=\left[1^{2} ;\left(21 ; 1^{4},\left(31^{2}\right)^{3}\right)^{4}\right]$, a proven 3-tower group $G \simeq\left\langle 3^{8}, \mathbf{6 2 9}\right\rangle-\# 1 ; 2-\# 2 ; 1 \mid 2$ of order $3^{11}$, and 3 -tower length $\ell_{3} K=3$,

- the unique field with discriminant $d=-91643$ has iterated IPAD of second order $\tau^{(2)} K=\left[1^{2} ;\left(21 ; 1^{4},\left(2^{3}\right)^{3}\right)^{2},\left(21 ; 1^{4},(321)^{3}\right)^{2}\right]$, unknown 3-tower group and 3-tower length $\ell_{3} K \geq 3$,

- the unique field with discriminant $d=-221944$ has iterated IPAD of second order $\tau^{(2)} K=\left[1^{2} ;\left(21 ; 1^{4},\left(3^{2} \mathbf{1}\right)^{3}\right)^{4}\right]$, but unknown 3-tower group and 3-tower length $\ell_{3} K \geq 3$.

Proof. Similar to the proof of Theorem 7.15, using Table 6, but now applied to the different range of discriminants and various iterated IPADs of second order.

\section{Imaginary Quadratic Fields of Type $(3,3,3)$ and Multi-Layered IPADs}

In the final section $\$ 7$ of [1], we proved that the second 3-class groups $\mathfrak{M}=\mathrm{G}_{3}^{2} K$ of the 14 imaginary quadratic fields $K=\mathbb{Q}(\sqrt{d})$ with fundamental discriminants $-10^{7}<d<0$ and 3-class group $\mathrm{Cl}_{3}(K)$ of type $(3,3,3)$ are pairwise non-isomorphic [[1], Thm. 7.1, p. 307]. For the proof of this theorem in [[1], $\$ 7.3$, p. 311], the IPADs of the 14 fields were not sufficient, since the three fields with discriminants

$$
d \in\{-4447704,-5067967,-8992363\}
$$

share the common accumulated (unordered) IPAD

$$
\tau^{(1)} K=\left[\tau_{0} K ; \tau_{1} K\right]=\left[1^{3} ; 32^{2} 1 ;\left(21^{4}\right)^{5},\left(2^{2} 1^{2}\right)^{7}\right] .
$$

To complete the proof we had to use information on the occupation numbers of the accumulated (unordered) IPODs,

$$
\varkappa_{1} K=\left[1,2,6,(8)^{6}, 9,(10)^{2}, 13\right] \text { with maximal occupation number } 6 \text { for }
$$
$d=-4447704$,

$\varkappa_{1} K=\left[1,2,(3)^{2},(4)^{2}, 6,(7)^{2}, 8,(9)^{2}, 12\right]$ with maximal occupation number 2 for $d=-5067967$,

$\varkappa_{1} K=\left[(2)^{2}, 5,6,7,(9)^{2},(10)^{3},(12)^{3}\right]$ with maximal occupation number 3 for $d=-8992363$.

Meanwhile we succeeded in computing the second layer of the transfer target type, $\tau_{2} K$, for the three critical fields with the aid of the computational algebra system MAGMA [17] by determining the structure of the 3-class groups $\mathrm{Cl}_{3} L$ of the 13 unramified bicyclic bicubic extensions $L / K$ with relative degree $[L: K]=3^{2}$ and absolute degree 18. In accumulated (unordered) form the second layer of the TTT is given by 


$$
\begin{aligned}
\tau_{2} K & =\left[32^{5} 1^{2} ; 4321^{5} ; 2^{5} 1^{3},\left(3^{2} 21^{5}\right)^{2} ; 2^{4} 1^{4}, 32^{2} 1^{5} ;\left(2^{2} 1^{7}\right)^{3},\left(2^{3} 1^{5}\right)^{3}\right] \text { for } \\
d=-4447704 & \\
\tau_{2} K & =\left[3^{2} 2^{2} 1^{4} ;\left(3^{2} 21^{5}\right)^{3} ; 32^{2} 1^{5} ;\left(2^{3} 1^{5}\right)^{8}\right] \text { for } d=-5067967, \text { and } \\
\tau_{2} K & =\left[32^{2} 1^{6},\left(3^{2} 21^{5}\right)^{3} ; 2^{4} 1^{4}, 32^{2} 1^{5} ; 2^{2} 1^{7},\left(2^{3} 1^{5}\right)^{6}\right] \text { for } d=-8992363 .
\end{aligned}
$$

These results admit incredibly powerful conclusions, which bring us closer to the ultimate goal to determine the precise isomorphism type of $\mathrm{G}_{3}^{2} K$. Firstly, they clearly show that the second 3-class groups of the three critical fields are pairwise non-isomorphic without using the IPODs. Secondly, the component with the biggest order establishes an impressively sharpened estimate for the order of $\mathrm{G}_{3}^{2} \mathrm{~K}$ from below. The background is explained by the following lemma.

Lemma 8.1. Let $G$ be a finite p-group with abelianization $G / G^{\prime}$ of type $(p, p, p)$ and denote by $\operatorname{lo}_{p}(G):=\log _{p}(\operatorname{ord}(G))$ the logarithmic order of $G$ with respect to the prime number $p$. Then the abelianizations $H / H^{\prime}$ of subgroups $H<G$ in various layers of $G$ admit lower bounds for $\operatorname{lo}_{p}(G)$ :

1) $\operatorname{lo}_{p}(G) \geq 1+\max \left\{\operatorname{lo}_{p}\left(H / H^{\prime}\right) \mid H \in \operatorname{Lyr}_{1} G\right\}$.

2) $\operatorname{lo}_{p}(G) \geq 2+\max \left\{\operatorname{lo}_{p}\left(H / H^{\prime}\right) \mid H \in \operatorname{Lyr}_{2} G\right\}$.

3) $\operatorname{lo}_{p}(G) \geq 3+\operatorname{lo}_{p}\left(G^{\prime} / G^{\prime \prime}\right)$, and in particular we have an equation $\operatorname{lo}_{p}(G)=3+\operatorname{lo}_{p}\left(G^{\prime}\right)$ if $G$ is metabelian.

Proof. The Lagrange formula for the order of $G$ in terms of the index of a subgroup $H \leq G$ reads

$$
\operatorname{ord}(G)=(G: H) \cdot \operatorname{ord}(H),
$$

and taking the $p$-logarithm yields

$$
\operatorname{lo}_{p}(G)=\log _{p}((G: H))+\operatorname{lo}_{p}(H) .
$$

In particular, we have $\log _{p}((G: H))=\log _{p}\left(p^{n}\right)=n$ for $H \in \operatorname{Lyr}_{n} G$, $0 \leq n \leq 3$, and again by the Lagrange formula

$$
\operatorname{ord}(H)=\left(H: H^{\prime}\right) \cdot \operatorname{ord}\left(H^{\prime}\right) \geq\left(H: H^{\prime}\right),
$$

respectively

$$
\operatorname{lo}_{p}(H)=\log _{p}\left(\left(H: H^{\prime}\right)\right)+\operatorname{lo}_{p}\left(H^{\prime}\right) \geq \operatorname{lo}_{p}\left(H / H^{\prime}\right),
$$

with equality if and only if $H^{\prime}=1$, that is, $H$ is abelian.

Finally, $G$ is metabelian if and only if $G^{\prime}$ is abelian.

Let us first draw weak conclusions from the first layer of the TTT, i.e. the IPAD, with the aid of Lemma 8.1.

Theorem 8.1. (Coarse estimate [1])

The order of $\mathfrak{M}=\mathrm{G}_{3}^{2} K$ for the three critical fields $K$ is bounded from below by $\operatorname{ord}(\mathfrak{M}) \geq 3^{9}$. If the maximal subgroup $H<\mathfrak{M}$ with the biggest order of $H / H^{\prime}$ is abelian, i.e. $H^{\prime}=1$, then the precise logarithmic order of $\mathfrak{M}$ is given by $\mathrm{lo}_{3}(\mathfrak{M})=9$.

Proof. The three critical fields with discriminants 
$d \in\{-4447704,-5067967,-8992363\}$ share the common accumulated IPAD $\tau^{(1)} K=\left[\tau_{0} K ; \tau_{1} K\right]=\left[1^{3} ;\left(32^{2} 1 ;\left(21^{4}\right)^{5},\left(2^{2} 1^{2}\right)^{7}\right)\right]$.

Consequently, Lemma 8.1 yields a uniform lower bound for each of the three fields:

$\mathrm{lo}_{3}(\mathfrak{M}) \geq 1+\max \left\{\mathrm{lo}_{3}\left(H / H^{\prime}\right) \mid H \in \mathrm{Lyr}_{1} \mathfrak{M}\right\}=1+\mathrm{lo}_{3}\left(32^{2} 1\right)=1+3+2 \times 2+1=9$.

The assumption that a maximal subgroup $U<\mathfrak{M}$ having not the biggest order of $U / U^{\prime}$ were abelian (with $U / U^{\prime} \simeq U$ ) immediately yields the contradiction that

$$
\begin{aligned}
\operatorname{lo}_{3}(\mathfrak{M}) & =\log _{3}((\mathfrak{M}: U))+\mathrm{lo}_{3}(U)=1+\operatorname{lo}_{3}\left(U / U^{\prime}\right) \\
& <1+\max \left\{\operatorname{lo}_{3}\left(H / H^{\prime}\right) \mid H \in \operatorname{Lyr}_{1} \mathfrak{M}\right\} \leq \operatorname{lo}_{3}(\mathfrak{M}) .
\end{aligned}
$$

It is illuminating that much stronger estimates and conclusions are possible by applying Lemma 8.1 to the second layer of the TTT.

Theorem 8.2. (Finer estimates)

None of the maximal subgroups of $\mathfrak{M}=\mathrm{G}_{3}^{2} K$ for the three critical fields $K$ can be abelian.

The logarithmic order of $\mathfrak{M}$ is bounded from below by

$\mathrm{lo}_{3}(\mathfrak{M}) \geq 17$ for $d=-4447704$,

$\mathrm{lo}_{3}(\mathfrak{M}) \geq 16$ for $d=-5067967$,

$\mathrm{lo}_{3}(\mathfrak{M}) \geq 15$ for $d=-8992363$.

Proof. As mentioned earlier already, computations with MAGMA [17] have shown that the accumulated second layer of the TTT is given by

$$
\begin{aligned}
\tau_{2} K & =\left[32^{5} 1^{2} ; 4321^{5} ; 2^{5} 1^{3},\left(3^{2} 21^{5}\right)^{2} ; 2^{4} 1^{4}, 32^{2} 1^{5} ;\left(2^{2} 1^{7}\right)^{3},\left(2^{3} 1^{5}\right)^{3}\right] \text { for } \\
d=-4447704 & \\
\tau_{2} K & =\left[3^{2} 2^{2} 1^{4} ;\left(3^{2} 21^{5}\right)^{3} ; 32^{2} 1^{5} ;\left(2^{3} 1^{5}\right)^{8}\right] \text { for } d=-5067967, \text { and } \\
\tau_{2} K & =\left[32^{2} 1^{6},\left(3^{2} 21^{5}\right)^{3} ; 2^{4} 1^{4}, 32^{2} 1^{5} ; 2^{2} 1^{7},\left(2^{3} 1^{5}\right)^{6}\right] \text { for } d=-8992363 .
\end{aligned}
$$

Consequently the maximal logarithmic order

$$
\begin{aligned}
M & :=\max \left\{\operatorname{lo}_{3}\left(H / H^{\prime}\right) \mid H \in \operatorname{Lyr}_{2} \mathfrak{M}\right\} \text { is } \\
M & =\operatorname{lo}_{3}\left(32^{5} 1^{2}\right)=3+5 \times 2+2 \times 1=15 \text { for } d=-4447704, \\
M & =\operatorname{lo}_{3}\left(3^{2} 2^{2} 1^{4}\right)=2 \times 3+2 \times 2+4 \times 1=14 \text { for } d=-5067967, \\
M & =\operatorname{lo}_{3}\left(32^{2} 1^{6}\right)=3+2 \times 2+6 \times 1=13 \text { for } d=-8992363 .
\end{aligned}
$$

According to Lemma 8.1, we have

$\operatorname{lo}_{3}(\mathfrak{M}) \geq 2+\max \left\{\operatorname{lo}_{3}\left(H / H^{\prime}\right) \mid H \in \operatorname{Lyr}_{2} \mathfrak{M}\right\}=2+M$.

Finally, if one of the maximal subgroups of $\mathfrak{M}$ were abelian, then Theorem 8.1 would give the contradiction that $\mathrm{lo}_{3}(\mathfrak{M})=9$.

Unfortunately, it was impossible for any of the three critical fields $K$ to compute the third layer of the TTT, $\tau_{3} K$, that is the structure of the 3-class group of the Hilbert 3 -class field $F_{3}^{1} K$ of $K$, which is of absolute degree 54 . This would have given the precise order of the metabelian group 
$\mathfrak{M}=\mathrm{G}_{3}^{2} K=\mathrm{Gal}\left(\mathrm{F}_{3}^{2} K / K\right)$, according to Lemma 8.1, since

$\mathfrak{M}^{\prime}=\operatorname{Gal}\left(\mathrm{F}_{3}^{2} K / \mathrm{F}_{3}^{1} K\right) \simeq \mathrm{Cl}_{3}\left(\mathrm{~F}_{3}^{1} K\right)$.

We also investigated whether the complete iterated IPAD of second order, $\tau^{(2)} \mathfrak{M}$, is able to improve the lower bounds in Theorem 8.2 further. It turned out that, firstly none of the additional non-normal components of $\left(\tau_{1} H\right)_{H \in \mathrm{Ly} \mathrm{I}_{1} \mathfrak{M}}$ seems to have bigger order than the normal components of $\tau_{2} \mathfrak{M}$, and secondly, due to the huge 3-ranks of the involved groups, the number of required class group computations enters astronomic regions.

To give an impression, we show the results for five of the 13 maximal subgroups in the case of $d=-4447704$ :

$$
\begin{aligned}
& \tau^{(1)} H_{1}=\left[2^{2} 1^{2} ; 32^{5} 1^{2} ;\left(2^{3} 1^{5}\right)^{3} ;\left(3^{2} 21^{2}\right)^{3} ;\left(321^{4}\right)^{9},\left(32^{2} 1^{2}\right)^{24}\right], \text { with } 40 \text { components, } \\
& \tau^{(1)} H_{2}=\left[21^{4} ; 32^{5} 1^{2} ; 2^{5} 1^{3} ; 2^{4} 1^{4} ; 2^{2} 1^{7} ;\left(31^{6}\right)^{3},\left(321^{4}\right)^{33} ;\left(321^{2}\right)^{81}\right], \text { with } 121
\end{aligned}
$$

components,

$$
\tau^{(1)} H_{3}=\left[2^{2} 1^{2} ; 32^{5} 1^{2} ; 32^{2} 1^{5} ;\left(2^{2} 1^{7}\right)^{2} ;\left(321^{5}\right)^{3},\left(32^{2} 1^{3}\right)^{6},\left(3^{2} 21^{2}\right)^{3},\left(32^{2} 1^{2}\right)^{24}\right] \text {, with }
$$

40 comp.,

$\tau^{(1)} H_{4}=\left[32^{2} 1 ; 32^{5} 1^{2} ; 4321^{5} ;\left(3^{2} 21^{5}\right)^{2} ;\left(4321^{3}\right)^{6} ;\left(431^{4}\right)^{6},\left(3^{2} 21^{3}\right)^{6},\left(4321^{2}\right)^{9},\left(3^{3} 1^{2}\right)^{9}\right]$, 40 comp.,

$$
\tau^{(1)} H_{5}=\left[2^{2} 1^{2} ; 3^{2} 21^{5} ; 32^{2} 1^{5}, 2^{4} 1^{4} ; 2^{3} 1^{5} ;\left(321^{3}\right)^{36}\right] \text {, with } 40 \text { components. }
$$

\section{Acknowledgements}

We gratefully acknowledge that our research is supported by the Austrian Science Fund (FWF): P 26008-N25.

Sincere thanks are given to Michael R. Bush (Washington and Lee University, Lexington, VA) for making available numerical results on IPADs of real quadratic fields $K=\mathbb{Q}(\sqrt{d})$, and the distribution of discriminants $d<10^{9}$ over these IPADs [42].

We are indebted to Nigel Boston, Michael R. Bush and Farshid Hajir for kindly making available an unpublished database containing numerical results of their paper [13] and a related paper on real quadratic fields, which is still in preparation.

A succinct version of the present article has been delivered on July 09, 2015, within the frame of the 29ièmes Journées Arithmétiques at the University of Debrecen, Hungary [2].

\section{Funding}

Research supported by the Austrian Science Fund (FWF): P 26008-N25.

\section{References}

[1] Mayer, D.C. (2015) Index-p Abelianization Data of p-Class Tower Groups. Advances in Pure Mathematics, 5, 286-313.

[2] Mayer, D.C. (2015) Index-p Abelianization Data of p-Class Tower Groups. 29ièmes Journées Arithmétiques (JA 2015), Univ. of Debrecen, Hungary, Presentation Delivered on 9 July 2015. 
[3] Mayer, D.C. (2015) Periodic Sequences of p-Class Tower Groups. Journal of Applied Mathematics and Physics, 3, 746-756.

[4] Mayer, D.C. (2016) Artin Transfer Patterns on Descendant Trees of Finite p-Groups. Advances in Pure Mathematics, 6, 66-104.

[5] Mayer, D.C. (2012) The Second p-Class Group of a Number Field. International Journal of Number Theory, 8, 471-505.

[6] A. Scholz und O. Taussky (1934) Die Hauptideale der kubischen Klassenkörper imaginär quadratischer Zahlkörper: Ihre rechnerische Bestimmung und ihr Einfluß auf den Klassenkörperturm. Journal für die Reine und Angewandte Mathematik, 171, 19-41.

[7] Bush, M.R. and Mayer, D.C. (2015) 3-Class Field Towers of Exact Length 3. Journal of Number Theory, 147, 766-777. https://doi.org/10.1016/j.jnt.2014.08.010

[8] H. Koch und B. B. Venkov, (1975) Über den p-Klassenkörperturm eines imaginär-quadratischen Zahlkörpers. Asté risque, 24-25, 57-67.

[9] Artin, E. (1927) Beweis des allgemeinen Reziprozitätsgesetzes. Abhandlungen aus dem Mathematischen Seminar der Universität Hamburg, 5, 353-363. https://doi.org/10.1007/BF02952531

[10] Mayer, D.C. (2014) Quadratic p-Ring Spaces for Counting Dihedral Fields. International Journal of Number Theory, 10, 2205-2242.

[11] Artin, E. (1929) Idealklassen in Oberkörpern und allgemeines Reziprozitätsgesetz. Abhandlungen aus dem Mathematischen Seminar der Universität Hamburg, 7, 46-51. https://doi.org/10.1007/BF02941159

[12] Furtwängler, Ph. (1929) Beweis des Hauptidealsatzes für die Klassenkörper algebraischer Zahlkörper. Abhandlungen aus dem Mathematischen Seminar der Universität Hamburg, 7, 14-36. https://doi.org/10.1007/BF02941157

[13] Boston, N., Bush, M.R. and Hajir, F. (2014) Heuristics for p-Class Towers of Imaginary Quadratic fields. To Appear in Math. Annalen, 2016. (arXiv: 1111.4679v2 [math.NT] 10 Dec 2014.)

[14] The PARI Group, PARI/GP, Version 2.9.0, Bordeaux, 2016. http://pari.math.u-bordeaux.fr

[15] Bosma, W., Cannon, J. and Playoust, C. (1997) The Magma Algebra System. I. The User Language. Journal of Symbolic Computation, 24, 235-265. https://doi.org/10.1006/jsco.1996.0125

[16] Bosma, W., Cannon, J.J., Fieker, C. and Steels, A., Eds. (2016) Handbook of Magma Functions. Edition 2.22, Sydney.

[17] The MAGMA Group (2016) MAGMA Computational Algebra System. Version 2.22-6, Sydney. http://magma.maths.usyd.edu.au

[18] Mayer, D.C. (2012) Transfers of Metabelian p-Groups. Monatshefte für Mathematik, 166, 467-495.

[19] Taussky, O. (1932) Über eine Verschärfung des Haupidealsatzes für algebraische Zahlkörper. Journal für die Reine und Angewandte Mathematik, 168, 193-210.

[20] Mayer, D.C. and Newman, M.F. Finite 3-Groups with Transfer Kernel Type F. In Preparation.

[21] Mayer, D.C. (2016) p-Capitulation over Number Fields with p-Class Rank Two. Journal of Applied Mathematics and Physics, 4, 1280-1293.

[22] Mayer, D.C. (2015) New Number Fields with Known p-Class Tower. 22nd Czech and Slovak International Conference on Number Theory (CSICNT 2015), Liptovský Ján, Slovakia, Presentation Delivered on 31 August 2015. 
[23] Mayer, D.C. (2015) New Number Fields with Known p-Class Tower. Tatra Mountains Mathematical Publications, 64, 21-57.

[24] Mayer, D.C. (2014) Principalization Algorithm via Class Group Structure. Journal de Théorie des Nombres de Bordeaux, 26, 415-464.

[25] Besche, H.U., Eick, B. and O’Brien, E.A. (2002) A Millennium Project: Constructing Small Groups. International Journal of Algebra and Computation, 12, 623-644. https://doi.org/10.1142/S0218196702001115

[26] Besche, H.U., Eick, B. and O’Brien, E.A. (2005) The SmallGroups Library-A Library of Groups of Small Order. An Accepted and Refereed GAP Package, Available Also in MAGMA.

[27] Newman, M.F. Determination of Groups of Prime-Power Order. In: Group Theory, Canberra, 1975, Lecture Notes in Math., Vol. 573, Springer, Berlin, 1977, 73-84. https://doi.org/10.1007/bfb0087814

[28] O’Brien, E.A. (1990) The p-Group Generation Algorithm. Journal of Symbolic Computation, 9, 677-698. https://doi.org/10.1016/S0747-7171(08)80082-X

[29] Mayer, D.C. (2013) The Distribution of Second p-Class Groups on Coclass Graphs. Journal de Théorie des Nombres de Bordeaux, 25, 401-456.

[30] Mayer, D.C. (2015) Periodic Bifurcations in Descendant Trees of Finite p-Groups. Advances in Pure Mathematics, 5, 162-195.

[31] Gamble, G., Nickel, W. and O'Brien, E.A. (2006) ANU p-Quotient-p-Quotient and p-Group Generation Algorithms. An Accepted GAP Package, Available Also in MAGMA.

[32] The GAP Group (2016) GAP_Groups, Algorithms, and Programming-A System for Computational Discrete Algebra. Version 4.8.6, Aachen, Braunschweig, Fort Collins, St. Andrews. http://www.gap-system.org

[33] Mayer, D.C. (2011) The Distribution of Second p-Class Groups on Coclass Graphs. 27ìmes Journées Arithmétiques (JA 2011), Faculty of Mathematics and Informatics, Univ. of Vilnius, Lithuania, Presentation Delivered on 1 July 2011.

[34] Mayer, D.C. (2016) Three-Stage Towers of 5-Class Fields. arXiv: 1604.06930v1 [math.NT] 23 Apr 2016.

[35] Mayer, D.C. (2016) Recent Progress in Determining p-Class Field Towers. Gulf J. Math. (Dubai, UAE). arXiv: 1605.09617v1 [math.NT] 31 May 2016.

[36] Mayer, D.C. (2016) Recent Progress in Determining p-Class Field Towers. 1st International Colloquium of Algebra, Number Theory, Cryptography and Information Security (ANCI 2016), Taza, Morocco, Invited Keynote Delivered on 12 November 2016.

[37] Nebelung, B. (1989) Klassifikation metabelscher 3-Gruppen mit Faktorkommutatorgruppe vom Typ $(3,3)$ und Anwendung auf das Kapitulationsproblem. Inauguraldissertation, Universität zu Köln.

[38] Ascione, J.A., Havas, G. and Leedham-Green, C.R. (1977) A Computer Aided Classification of Certain Groups of Prime Power Order. Bulletin of the Australian Mathematical Society, 17, 257-274, Corrigendum 317-319, Microfiche Supplement, 320.

[39] Ascione, J.A. (1979) On 3-Groups of Second Maximal Class. Ph.D. Thesis, Australian National University, Canberra.

[40] F.-P. Heider und B. Schmithals, (1982) Zur Kapitulation der Idealklassen in unverzweigten primzyklischen Erweiterungen. Journal für die Reine und Angewandte Mathematik, 336, 1-25. 
[41] Mayer, D.C. (1991) List of Discriminants $d_{L}<200000$ of Totally Real Cubic Fields L, Arranged According to Their Multiplicities $\mathrm{m}$ and Conductors f. Computer Centre, Department of Computer Science, University of Manitoba, Winnipeg, Canada, Austrian Science Fund, Project Nr. J0497-PHY.

[42] Bush, M.R. (2015) IPADs of Real Quadratic Fields with 3-Class Rank Two and Discriminants up to $10^{9}$. 11 July 2015, Private Communication.

[43] Blackburn, N. (1957) On Prime-Power Groups in Which the Derived Group Has Two Generators. Proceedings of the Cambridge Philosophical Society, 53, 19-27. https://doi.org/10.1017/S0305004100031959

[44] Shafarevich, I.R. (1964) Extensions with Prescribed Ramification Points (Russian). Publications Mathématiques, Institut des Hautes Études Scientiques, 18, 71-95. (English transl. by J. W. S. Cassels in American Mathematical Society Translations, II. Series, 59 (1966), 128-149.)

[45] Bartholdi, L. and Bush, M.R. (2007) Maximal Unramified 3-Extensions of Imaginary Quadratic Fields and $\mathrm{SL}_{2} \mathbb{Z}_{3}$. Journal of Number Theory, 124, 159-166. https://doi.org/10.1016/j.jnt.2006.08.008

[46] Fieker, C. (2001) Computing Class Fields via the Artin Map. Mathematics of Computation, 70, 1293-1303.

[47] Mayer, D.C. (1991) Principalization in Complex $S_{3}$-Fields. Congressus Numerantium 80, 73-87. (Proceedings of the Twentieth Manitoba Conference on Numerical Mathematics and Computing, Univ. of Manitoba, Winnipeg, Canada, 1990.)

[48] Scholz, A. (1933) Idealklassen und Einheiten in kubischen Körpern. Monatshefte für Mathematik und Physik, 40, 211-222. 


\section{Appendix: Corrigenda in [5] [24] [29]}

1) The restriction of the numerical results in Proposition 7.1 to the range $0<d<10^{7}$ is in perfect accordance with our machine calculations by means of PARI/GP [14] in 2010, and thus provides the first independent verification of data in [5] [24] [29].

However, in the manual evaluation of this extensive data material for the ground state of the types a.1, a.2, a.3, and a. $3^{*}$, a few errors crept in, which must be corrected at three locations: in the tables [[5], Tbl. 2, p. 496] and [[24], Tbl. 6.1, p. 451], and in the tree diagram [[29], Fig. 3.2, p. 422].

The absolute frequency of the ground state is actually given by

1382 instead of the incorrect 1386 for the union of types a.2 and a.3,

698 instead of the incorrect 697 for type a. $3^{*}$,

2080 instead of the incorrect 2083 for the union of types a.2, a.3, and a.3* and

150 instead of the incorrect 147 for type a. 1 .

(The three discriminants $d \in\{7643993,7683308,8501541\}$ were erroneously classified as type a.2 or a.3 instead of a.1.)

In the second table, two relative frequencies (percentages) should be updated:

$$
\begin{aligned}
& \frac{1382}{2303} \approx 60.0 \% \text { instead of } \frac{1386}{2303} \approx 60.2 \% \text { and } \\
& \frac{698}{2303} \approx 30.3 \% \text { instead of } \frac{697}{2303} \approx 30.3 \% .
\end{aligned}
$$

2) Incidentally, although it does not concern the section a of IPODs, the single field with discriminant $d=2747001$ was erroneously classified as type c.18, $\varkappa_{1}=(0313)$, instead of H.4, $\varkappa_{1}=(3313)$. This has consequences at four locations: in the tables [[5], Tbl. 4-5, pp. 498-499] and [[24], Tbl. 6.5, p. 452], and in the tree diagram [[29], Fig. 3.6, p. 442].

The absolute frequency of these types is actually given by

28 instead of the incorrect 29 for type c.18 (see also [23], Prop. 7.2]),

4 instead of the incorrect 3 for type H.4.

In the first two tables, the total frequencies should be updated, correspondingly:

207 instead of the incorrect 206 in [[5], Tbl. 4, p. 498],

66 instead of the incorrect 67 in [[5], Tbl. 5, p. 499]. 
Submit or recommend next manuscript to SCIRP and we will provide best service for you:

Accepting pre-submission inquiries through Email, Facebook, LinkedIn, Twitter, etc. A wide selection of journals (inclusive of 9 subjects, more than 200 journals)

Providing 24-hour high-quality service

User-friendly online submission system

Fair and swift peer-review system

Efficient typesetting and proofreading procedure

Display of the result of downloads and visits, as well as the number of cited articles Maximum dissemination of your research work

Submit your manuscript at: http://papersubmission.scirp.org/

Or contact apm@scirp.org 